\title{
COVID-19 is affecting elderly people more and increasing in its severity
}

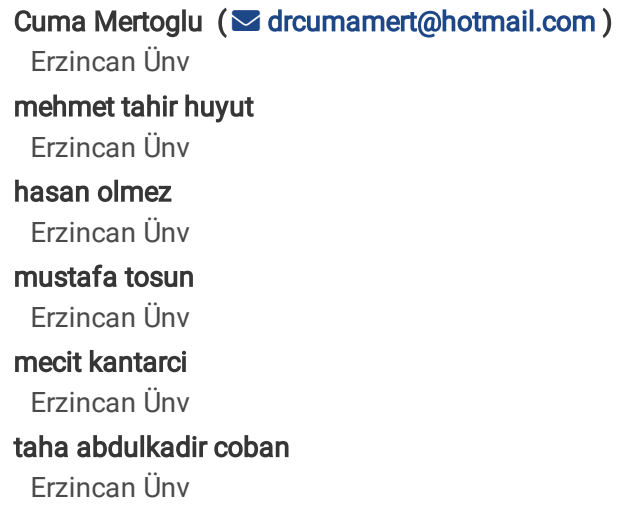

Research Article

Keywords: Corona Virus Disease 2019, SARS-CoV-2, blood biomarkers, seasonal changes

Posted Date: May 11th, 2021

DOI: https://doi.org/10.21203/rs.3.rs-512565/v1

License: (c) (i) This work is licensed under a Creative Commons Attribution 4.0 International License. Read Full License 


\section{Abstract \\ Objective}

The important changes occur in routine blood tests in Corona Virus Disease 2019 (COVID-19).

\section{Methods}

The biochemical, hematological and inflammatory biomarkers of a total of 3969 COVID-19 patients' results (3746 in the non-intensive care unit (non-ICU) group and 223 in the ICU group) were analyzed by dividing into three groups as spring, summer and autumn.

\section{Results}

In the non-ICU group, lymphocyte to monocyte ratio were lower in autumn than the other two seasons and neutrophil to lymphocyte ratio was higher in autumn than the other two seasons. Also, monocyte and platelet were higher in spring than autumn and eosinophil, hematocrit, hemoglobin, lymphocyte and red blood cells decreased from spring to autumn. In the non-ICU group, alanine aminotransferase and gamma-glutamyltransferase gradually increased from spring to autumn while albumin, alkaline phosphatase, calcium, total bilirubin and total protein gradually decreased. Additionally, C-reactive protein was higher in autumn than the other seasons, erythrocyte sedimentation rate was higher than the only summer.

\section{Conclusion}

The change in routine blood biomarkers in COVID-19 varies in the period from the emergence of the disease until now. Also, the timely change of blood biomarkers was mostly more negative and this indicates that the disease progresses more severely.

\section{Key Points}

- COVID-19 is affecting elderly people more.

- The changes, in routine blood biomarkers in COVID-19, varies in the period from the emergence of the disease until now.

- The timely changes of blood biomarkers were mostly more negative and this indicates that the disease progresses more severely.

- It was determined that how much the increases and decreases in the routine laboratory tests of the patients with COVID-19 changed based on the reference range.

\section{Introduction}

The coronavirus disease 2019 (COVID-19) caused by severe acute respiratory syndrome - coronavirus 2 (SARS-CoV-2), which emerged in Wuhan, China in December 2019 and was declared as a pandemic by the WHO on March 11th, 2020, continues to spread. A total of 93.805 .612 confirmed cases were detected in more than 200 countries until now and the disease caused the death of 2.026 .093 people [1]. It was reported that climate and seasonal changes have important effects on COVID-19 [2-5]. On the other hand, important mutations have occurred in the genetic structure of SARS-CoV-2 in time [6, 7].

Many studies have shown that there have been important changes in the routine laboratory test in COVID-19 [8-13]. However, most of these studies include data from the early period of the disease. The changes in the structure of the virus and the clinical characteristics of the disease within the process or seasonal changes may have caused routine laboratory tests to change. Thus, this study examined the changes in routine laboratory tests by seasons by constituting three periods of three months as of the emergence of COVID-19 disease. This study also examined how much the increases and decreases in the routine laboratory tests of the patients changed based on the reference range.

\section{Materials And Methods}

This retrospective single-center study was conducted by examining the records between March 10th, 2020, and November 30 th, 2020 from the laboratory information system of the hospital. Only adults older than 18 years were included in the study. Informed consent wasn't obtained because of this is a retrospective study. Local etic committee approved the study (date, 31 December 2020 and number, 2020-12-29T01). The diagnose of "U07.3 COVID-19 identified virus" was searched with indicated parameters and a total of 3969 patients (3746 in non-intensive care unit (non-ICU) group, 223 in ICU group) tests results were found. The diagnosis of COVID-19 was only made with a real-time polymerase chain reaction test and SARS CoV-2 virus nasal and pharyngeal swab specimens in the hospital during the period when the study was conducted. The patients in the intensive care unit were included in the ICU group and the patients in other patient wards and clinics were included in the non-ICU group and their laboratory data were compared in three month periods. The results between March 10th, 2020 and May 31st, 2020 were grouped as spring, the results between June 1st, 2020 and August 31 st, 2020 were grouped as summer, and the results between September 1st, 2020 and November 30st 2020 were grouped as autumn. The test results obtained from the first measurements of the patients recorded in the system were evaluated in this study.

The whole blood count was measured with Sysmex XN-1000 Hematology System (Sysmex Corporation, Kobe, Japan) autoanalyzer, and biochemical tests were made with the spectrophotometric method from serum in the Beckman Coulter Olympus AU2700 Plus Chemistry Analyzer (Beckman Coulter, Tokyo, Japan) autoanalyzer. Ferritin was measured by on sera chemiluminescence immunoassay (Centaur XP, Siemens Healthcare, Germany). Prothrombin time 
(PT), activated partial prothrombin time (aPTT) and fibrinogen on plasma was measured with a fully automated photo-optical coagulation instrument Ceveron-Alpha (Diapharma Group Inc. West Chester, Canada). C-reactive protein (CRP) was measured on serum by nephelometric method on BN ${ }^{\mathrm{TM}}$ II System (Siemens, Munich, Germany). Procalcitonin, D-dimer, and Troponin I were measured from the whole blood with AQT90 flex Radiometer® (Bronshoj, Denmark) device. The erythrocyte sedimentation rate (ESR) was analyzed on whole blood using TEST 1 BCL device (Alifax, Padova, Italy) based on the principle of photometric capillary flow kinetic analysis.

Neutrophil to lymphocyte ratio (NLR), calculated from a routine blood test by dividing absolute neutrophil count by absolute lymphocyte count, lymphocyte to monocyte ratio (LMR) calculated by dividing absolute lymphocyte count by monocyte count and platelet to lymphocyte ratio (PLR) calculated by dividing absolute platelet count by lymphocyte count. Derived NLR ratio (d-NLR); was calculated neutrophil count divided by the result of white blood cell (WBC) count minus neutrophil count). lymphocyte to C-reactive protein ratio (LCR); lymphocyte/C-reactive protein (CRP), systemic immune-inflammation index (SII); was calculated using the following formula: platelet*neutrophil/lymphocyte. The atherogenic index of plasma (AIP) is the logarithmically transformed ratio of triglycerides and high-density lipoprotein cholesterol (HDL-C). The values of the group with increased biomarker levels were divided into the upper threshold of the reference range and the values of the group with decreased biomarker levels were divided into the lower threshold of the reference range; thus, how much they changed was determined.

\section{Statistical analysis}

The one-way ANOVA test was used when the data were normally distributed in the three groups comparisons, otherwise, the Kruskal Wallis Test was carried out. An independent t-test or Mann-Whitney test was carried out to evaluate the continuous variables between any two groups. Using the $\chi 2$ test, proportions for categorical variables have been compared. All statistical analyses have been conducted by using SPSS software (version 20.0, SPSS Inc, Chicago, United States). Statistical significance can be shown as the $\mathrm{p}$-values $<0.05$.

\section{Results}

In the non-ICU group, the ratio of men to women was higher in spring while the ratio of women to men was higher in summer and autumn. The ratio of men was always higher in the ICU group. The mean age in the non-ICU group gradually increased from spring to autumn and this result was also similar in the ICU group. In the non-ICU group, basophil count was higher in spring than other two seasons, mean corpuscular hemoglobin ( $\mathrm{MCH}$ ) value was only higher in spring than autumn, and mean corpuscular hemoglobin concentration ( $\mathrm{MCHC}$ ), neutrophil, platelet distribution width (PDW) and red cell distribution width (RDW) values were higher in autumn than spring and higher in spring than summer. Additionally, mean corpuscular volume (MCV), platelet large cell ratio ( $\mathrm{P}$-LRC) and lymphocyte to monocyte ratio (LMR) values were lower in autumn than other two seasons, monocyte, platelet and white blood cell (WBC, in women) were higher in spring than autumn and higher in autumn than summer while mean platelet volume (MPV), NLR and dNLR values were higher in autumn than other seasons, and PCT and WBC (in men) were lower in summer than other seasons. Moreover, PLR value gradually increased from spring to autumn while eosinophil, hematocrit, hemoglobin, lymphocyte and red blood cells (RBC) values gradually decreased (Table 1). In the ICU group, MCHC was higher in autumn than in other seasons, monocyte was higher in spring than autumn while neutrophil, WBC (in women), NLR, dNLR and PLR were lower in summer than autumn. Age, MCV, neutrophil, plateletcrit, PDW, RDW, WBC, P-LRC, NLR, d-NLR and PLR values were higher and eosinophil, hematocrit, hemoglobin, lymphocyte, MCHC, monocyte, RBC, WBC and LMR were lower in the ICU group compared to the non-ICU group (Table 2). 
Seasonal comparison of demographical characteristics anc

\begin{tabular}{|c|c|c|c|c|c|c|c|c|c|c|c|c|c|c|}
\hline & \multicolumn{14}{|l|}{ Non-ICU } \\
\hline & \multicolumn{5}{|l|}{ Spring } & \multicolumn{5}{|l|}{ Summer } & \multicolumn{4}{|l|}{ Autumn } \\
\hline Male n (\%) & \multicolumn{5}{|c|}{$251(\%$ 61.1) } & \multicolumn{5}{|c|}{$492(\%$ 49.5) } & \multicolumn{4}{|c|}{$1125(\%$ 48.0) } \\
\hline Female $\mathrm{n}(\%)$ & \multicolumn{5}{|c|}{$160(\% 38.9)$} & \multicolumn{5}{|c|}{$501(\%$ 50.5) } & \multicolumn{4}{|c|}{$1217(\% 52.0)$} \\
\hline Test & Mean & Med & Min & Max & SD & Mean & Med & Min & Max & SD & Mean & Med & Min & Max \\
\hline Age (years) & $48^{*}$ & 46 & 19 & 96 & 19 & $53^{\llbracket}$ & 54 & 18 & 94 & 19 & $58^{\#}$ & 60 & 18 & 102 \\
\hline $\begin{array}{l}\text { BASO } \times 10^{\wedge} 3 / \\
\mu \mathrm{L}\end{array}$ & $0.04^{\#}$ & 0.04 & 0.01 & 0.17 & 0.02 & $0.03^{*}$ & 0.02 & 0.01 & 0.13 & 0.02 & $0.03^{*}$ & 0.02 & 0.01 & 0.38 \\
\hline $\begin{array}{l}\text { EOS } \times 10^{\wedge} 3 / \\
\mu \mathrm{L}\end{array}$ & $0.17^{\star}$ & 0.13 & 0.01 & 1.03 & 0.14 & $0.12^{\bigotimes}$ & 0.07 & 0.01 & 4.41 & 0.20 & $0.09^{\#}$ & 0.06 & 0.01 & 0.97 \\
\hline HCT (\%) & $41.38^{*}$ & 41.80 & 17.20 & 51.70 & 4.81 & $40.44^{\neq}$ & 40.60 & 22.90 & 53.80 & 5.19 & $38.62^{\rrbracket}$ & 38.60 & 12.00 & 56.30 \\
\hline $\mathrm{HGB}(\mathrm{g} / \mathrm{L})$ & $13.75^{\square}$ & 13.90 & 5.50 & 17.80 & 1.83 & $13.34^{\#}$ & 13.40 & 7.20 & 19.00 & 1.94 & $13.13^{*}$ & 13.10 & 4.00 & 18.60 \\
\hline $\begin{array}{l}\operatorname{LYM} M \times 10^{\wedge} 3 / \\
\mu \mathrm{L}\end{array}$ & $2.05^{*}$ & 1.98 & 0.49 & 11.91 & 0.87 & $1.67^{\square}$ & 1.57 & 0.09 & 7.55 & 0.74 & $1.51^{\#}$ & 1.33 & 0.08 & 53.96 \\
\hline $\mathrm{MCH}(\mathrm{mg})$ & $28.49^{\#}$ & 28.80 & 18.40 & 37.70 & 2.31 & $28.26^{\#^{*}}$ & 28.70 & 17.70 & 41.90 & 2.48 & $28.18^{*}$ & 28.50 & 15.60 & 39.90 \\
\hline $\mathrm{MCHC}(\mathrm{g} / \mathrm{dL})$ & $33.18^{\#}$ & 33.30 & 28.30 & 36.70 & 1.39 & $32.93^{*}$ & 33.00 & 27.10 & 37.10 & 1.38 & $33.95^{\square}$ & 34.10 & 3.60 & 38.40 \\
\hline $\operatorname{MCV}(f L)$ & $85.80^{\unrhd}$ & 85.90 & 61.00 & 117.80 & 5.37 & $85.74^{\bigotimes}$ & 86.20 & 58.20 & 116.20 & 5.93 & $82.92^{*}$ & 83.20 & 55.80 & 116.80 \\
\hline $\begin{array}{l}\text { MONOx10^3/ } \\
\mu \mathrm{L}\end{array}$ & $0.61^{*}$ & 0.57 & 0.13 & 4.20 & 0.29 & $0.50^{\rrbracket}$ & 0.47 & 0.03 & 2.19 & 0.22 & $0.53^{\#}$ & 0.50 & 0.05 & 6.29 \\
\hline MPV (fL) & $10.27^{\#}$ & 10.10 & 8.30 & 13.60 & 0.95 & $10.38^{\#}$ & 10.30 & 8.10 & 15.00 & 0.90 & $10.47^{\square}$ & 10.40 & 8.10 & 14.20 \\
\hline $\begin{array}{l}\mathrm{NEU} \times 10^{\wedge} 3 / \\
\mu \mathrm{L}\end{array}$ & $4.23^{\llbracket}$ & 3.82 & 0.68 & 15.87 & 1.95 & $3.69^{\#}$ & 3.23 & 0.49 & 26.73 & 2.15 & $4.80^{\llbracket}$ & 4.04 & 0.37 & 20.00 \\
\hline P.LCR (\%) & $26.96^{\text {【 }}$ & 26.10 & 11.90 & 51.60 & 7.54 & $27.94^{\bigotimes}$ & 27.30 & 11.10 & 61.30 & 7.22 & $28.92^{*}$ & 28.20 & 9.50 & 56.10 \\
\hline РСТ (\%) & $0.25^{\star}$ & 0.23 & 0.06 & 0.63 & 0.08 & $0.22^{\square}$ & 0.22 & 0.06 & 0.55 & 0.07 & $0.24^{*}$ & 0.23 & 0.01 & 1.22 \\
\hline PDW (fL) & $11.78^{*}$ & 11.40 & 8.00 & 22.30 & 2.21 & $11.90^{\text {*区 }}$ & 11.60 & 8.20 & 23.50 & 2.12 & $12.06^{\square}$ & 11.70 & 7.60 & 25.30 \\
\hline $\begin{array}{l}\mathrm{PLT} \times 10^{\wedge} 3 / \\
\mu \mathrm{L}\end{array}$ & $241.14^{\bigotimes}$ & 226.00 & 53.00 & 652.00 & 78.47 & $218.88^{\star}$ & 208.00 & 49.00 & 603.00 & 76.78 & $231.24^{\#}$ & 221.00 & 5.00 & 1199.0 \\
\hline $\begin{array}{l}\mathrm{RBC} \times 10^{\wedge} 6 / \\
\mu \mathrm{L}\end{array}$ & $4.84^{\#}$ & 4.85 & 1.46 & 6.16 & 0.58 & $4.73^{*}$ & 4.75 & 2.22 & 7.04 & 0.62 & $4.67^{\square}$ & 4.65 & 1.24 & 7.22 \\
\hline RDW (\%) & $13.37^{\mathbb{}}$ & 12.90 & 11.20 & 24.20 & 1.74 & $13.55^{\mathbb{Q}^{\star}}$ & 13.00 & 11.20 & 24.20 & 1.77 & $13.52^{*}$ & 13.20 & 11.00 & 27.00 \\
\hline Male & $7.2^{\#}$ & 6.8 & 2.8 & 18.9 & 2.2 & $6.4^{\rrbracket}$ & 6.1 & 1.0 & 19.0 & 2.5 & $7.5^{\#}$ & 6.8 & 0.4 & 60.2 \\
\hline $\begin{array}{l}\text { WBC } \times 10^{\wedge} 3 / \\
\mu \mathrm{L}\end{array}$ & $6.9^{\#}$ & 6.8 & 2.9 & 16.8 & 2.1 & $5.5^{\rrbracket}$ & 5.2 & 1.3 & 36.6 & 2.5 & $6.3^{*}$ & 5.9 & 1.4 & 24.0 \\
\hline NLR & $2.49^{\star}$ & 1.93 & 0.06 & 17.56 & 1.94 & $2.80^{\star}$ & 1.92 & 0.29 & 74.67 & 3.41 & $4.95^{\llbracket}$ & 2.80 & 0.07 & 93.08 \\
\hline PLR & $134.15^{\llbracket}$ & 116.56 & 15.79 & 708.11 & 71.23 & $154.68^{*}$ & 130.77 & 18.22 & 1322.22 & 96.67 & $210.52^{\#}$ & 156.83 & 2.74 & 2287.5 \\
\hline $\mathrm{dNLR}$ & $1.65^{\#}$ & 1.39 & 0.04 & 7.82 & 1.00 & $1.92^{\#}$ & 1.40 & 0.26 & 84.00 & 2.98 & $2.97^{\star}$ & 1.94 & 0.07 & 33.09 \\
\hline LMR & $3.66^{*}$ & 3.55 & 0.73 & 12.62 & 1.51 & $3.74^{*}$ & 3.55 & 0.72 & 12.90 & 1.77 & $3.17^{\#}$ & 2.84 & 0.12 & 47.69 \\
\hline
\end{tabular}

*, \#, Mean values with the same sign are similar, those with different signs have a statistically significant difference. ${ }^{*} p<0.05,{ }^{* *} p<0.01,{ }^{* *} p<0.001, p$ value: ratio; LYM, lymphocyte; Max, maximum; MCH, mean corpuscular hemoglobin; MCHC, mean corpuscular hemoglobin concentration; MCV, mean corpuscular v LRC, platelet large cell ratio; PLR, platelet to lymphocyte ratio; PLT, platelet; RBC, red blood cells; RDW, red cell distribution width; SD, standard deviation; WBC, 
Table 2

Comparison of demographical characteristics and hematological biomarkers in the intensive care unit (ICU) and non-ICU groups with coronavirus disease 2019 on admission.

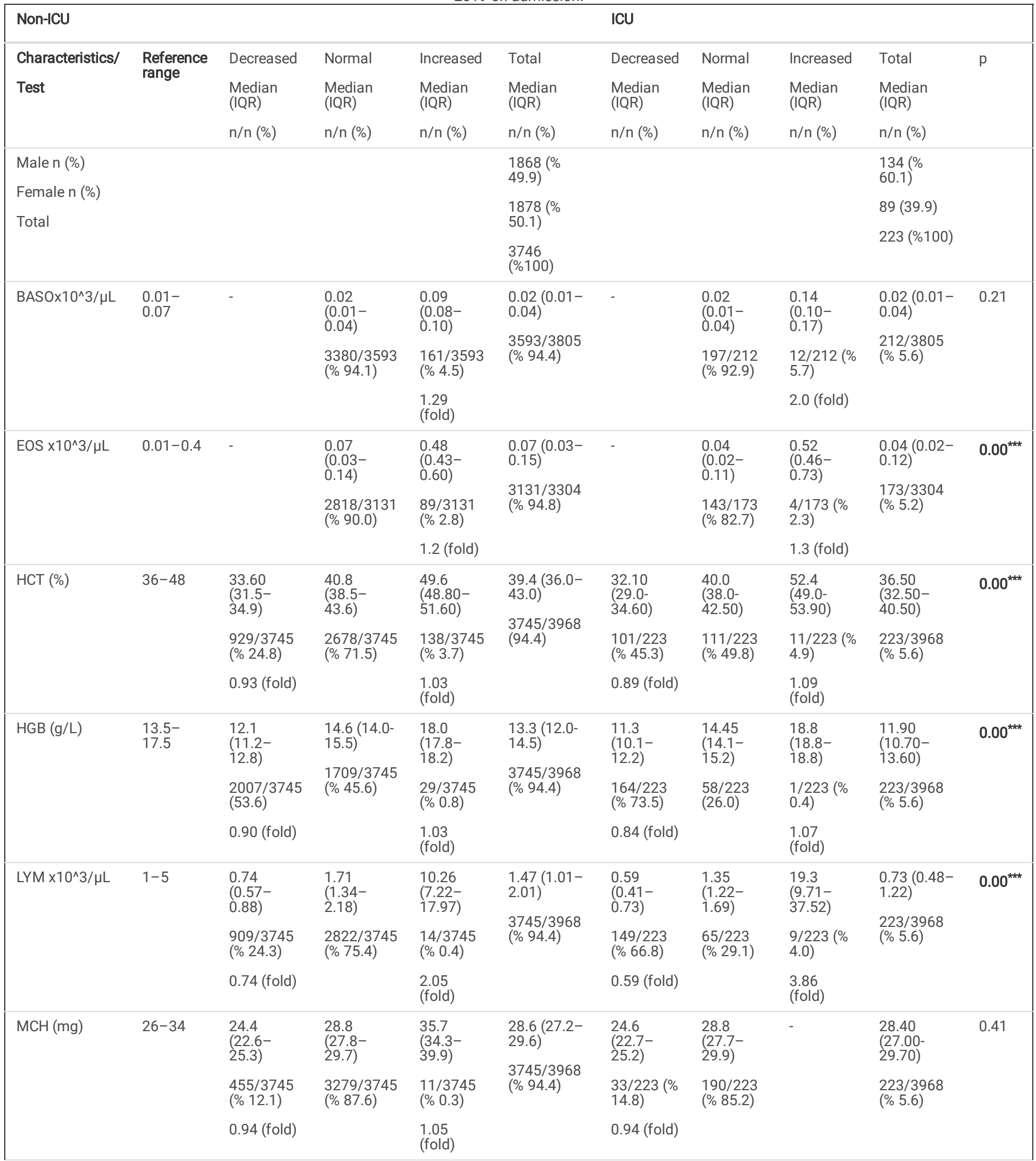

${ }^{*} \mathrm{p}<0.05,{ }^{* *} \mathrm{p}<0.01,{ }^{* * *} \mathrm{p}<0.001, \mathrm{p}$ values denoted comparison of total median values between ICU and non-ICU groups. BASO, basophil; d-NLR, derived neutrophil to lymphocyte ratio; EOS, eosinophil; HCT, hematocrit; HGB, hemoglobin; IQR, interquartile range; LMR, lymphocyte to monocyte ratio; LYM, lymphocyte; $\mathrm{MCH}$, mean corpuscular hemoglobin; $\mathrm{MCHC}$, mean corpuscular hemoglobin concentration; MCV, mean corpuscular volume; MONO, monocyte; MPV, mean platelet volume; NEU, neutrophil; NLR, neutrophil to lymphocyte ratio; PCT, plateletcrit; PDW, platelet distribution width; P-LRC, platelet large cell ratio; PLR, platelet to lymphocyte ratio; PLT, platelet; RBC, red blood cells; RDW, red cell distribution width; SD, standard deviation; WBC, white blood cell. 


\begin{tabular}{|c|c|c|c|c|c|c|c|c|c|c|}
\hline Non-ICU & & & & & & ICU & & & & \\
\hline \multirow[t]{4}{*}{$\mathrm{MCHC}(\mathrm{g} / \mathrm{dL})$} & \multirow[t]{4}{*}{$31-36$} & $\begin{array}{l}29.7 \\
(29.2- \\
30.0)\end{array}$ & $\begin{array}{l}33.7 \\
(32.8- \\
34.5)\end{array}$ & $\begin{array}{l}36.4 \\
(36.2- \\
36.7)\end{array}$ & \multirow[t]{3}{*}{$\begin{array}{l}33.7(32.7- \\
34.6)\end{array}$} & $\begin{array}{l}29.5 \\
(28.4- \\
29.9)\end{array}$ & $\begin{array}{l}33.3 \\
(32.6- \\
34.1)\end{array}$ & $\begin{array}{l}36.7 \\
(36.2- \\
37.2)\end{array}$ & $\begin{array}{l}33.00 \\
(31.50- \\
34.00)\end{array}$ & \multirow[t]{4}{*}{$0.00^{\star \star *}$} \\
\hline & & $\begin{array}{l}81 / 3660 \\
(\% 2.2)\end{array}$ & \multirow[t]{3}{*}{$\begin{array}{l}3446 / 3660 \\
(\% 94.2)\end{array}$} & $\begin{array}{l}133 / 3660 \\
(3.6)\end{array}$ & & $\begin{array}{l}25 / 209(\% \\
12.0)\end{array}$ & $\begin{array}{l}179 / 209 \\
(\% 85.6)\end{array}$ & $\begin{array}{l}5 / 209(\% \\
2.4)\end{array}$ & \multirow[t]{3}{*}{$\begin{array}{l}209 / 3869 \\
(\% 5.4)\end{array}$} & \\
\hline & & 0.96 (fold) & & $\begin{array}{l}1.01 \\
\text { (fold) }\end{array}$ & & 0.95 (fold) & & $\begin{array}{l}1.02 \\
\text { (fold) }\end{array}$ & & \\
\hline & & & & & $\begin{array}{l}3660 / 3869 \\
(\% 94.6)\end{array}$ & & & & & \\
\hline \multirow[t]{3}{*}{$\mathrm{MCV}(\mathrm{fL})$} & \multirow[t]{3}{*}{$80-96$} & $\begin{array}{l}77.2 \\
(74.3- \\
78.9)\end{array}$ & \multirow{3}{*}{$\begin{array}{l}85.3(83.0- \\
87.9) \\
3009 / 3745 \\
(\% 80.3)\end{array}$} & $\begin{array}{l}99.4 \\
(96.8- \\
102.6)\end{array}$ & \multirow{3}{*}{$\begin{array}{l}84.3(81.1- \\
87.3) \\
3745 / 3968 \\
(\% 94.4)\end{array}$} & $\begin{array}{l}77.2 \\
(73.4- \\
78.1)\end{array}$ & $\begin{array}{l}86.5 \\
(83.4- \\
90.0)\end{array}$ & $\begin{array}{l}99.75 \\
(97.6- \\
104.3)\end{array}$ & \multirow{3}{*}{$\begin{array}{l}86.10 \\
(81.70- \\
90.20) \\
223 / 3968 \\
(\% 5.6)\end{array}$} & \multirow[t]{3}{*}{$0.00^{\star \star * *}$} \\
\hline & & $\begin{array}{l}697 / 3745 \\
(\% 18.6)\end{array}$ & & $\begin{array}{l}39 / 3745 \\
(\% 1.0)\end{array}$ & & $\begin{array}{l}34 / 223(\% \\
15.2)\end{array}$ & $\begin{array}{l}177 / 223 \\
(\% 79.4)\end{array}$ & $\begin{array}{l}12 / 223(\% \\
5.4)\end{array}$ & & \\
\hline & & 0.97 (fold) & & $\begin{array}{l}1.04 \\
\text { (fold) }\end{array}$ & & 0.97 (fold) & & $\begin{array}{l}1.04 \\
\text { (fold) }\end{array}$ & & \\
\hline \multirow[t]{3}{*}{ MONO (\%) } & \multirow[t]{3}{*}{$0.1-1$} & $\begin{array}{l}0.08 \\
(0.06- \\
0.09)\end{array}$ & $\begin{array}{l}0.49 \\
(0.37- \\
0.63)\end{array}$ & $\begin{array}{l}1.14 \\
(1.06- \\
1.28)\end{array}$ & \multirow{3}{*}{$\begin{array}{l}0.50(0.37- \\
0.65) \\
3745 / 3968 \\
(\% 94.4)\end{array}$} & $\begin{array}{l}0.08 \\
(0.06- \\
0.09)\end{array}$ & $\begin{array}{l}0.42 \\
(0.30- \\
0.59)\end{array}$ & $\begin{array}{l}1.24 \\
(1.10- \\
1.35)\end{array}$ & \multirow{3}{*}{$\begin{array}{l}0.44(0.30- \\
0.66) \\
223 / 3968 \\
(\% 5.6)\end{array}$} & \multirow[t]{3}{*}{$0.00^{* *}$} \\
\hline & & $\begin{array}{l}29 / 3745 \\
(\% 0.8)\end{array}$ & $\begin{array}{l}3559 / 3745 \\
(\% 95.0)\end{array}$ & $\begin{array}{l}157 / 3745 \\
(\% 4.2)\end{array}$ & & $\begin{array}{l}7 / 223(\% \\
3.1)\end{array}$ & $\begin{array}{l}194 / 223 \\
(\% 87.0)\end{array}$ & $\begin{array}{l}22 / 223(\% \\
9.9)\end{array}$ & & \\
\hline & & 0.8 (fold) & & $\begin{array}{l}1.14 \\
\text { (fold) }\end{array}$ & & 0.8 (fold) & & $\begin{array}{l}1.24 \\
\text { (fold) }\end{array}$ & & \\
\hline \multirow[t]{3}{*}{ MPV (fL) } & \multirow[t]{3}{*}{$9.1-11.9$} & \multirow{2}{*}{$\begin{array}{l}8.80(8.70- \\
9.00) \\
159 / 3692 \\
(\% 4.3)\end{array}$} & \multirow{3}{*}{$\begin{array}{l}10.30 \\
(9.8-10.9) \\
3302 / 3692 \\
(\% 89.4)\end{array}$} & $\begin{array}{l}12.4 \\
(12.1- \\
12.8)\end{array}$ & \multirow{3}{*}{$\begin{array}{l}10.3(9.8- \\
11.0) \\
3692 / 3906 \\
(\% 94.5)\end{array}$} & $\begin{array}{l}8.80 \\
(8.60- \\
8.90)\end{array}$ & $\begin{array}{l}10.7 \\
(10.3- \\
11.3)\end{array}$ & $\begin{array}{l}12.9 \\
(12.3- \\
13.4)\end{array}$ & \multirow{3}{*}{$\begin{array}{l}10.90 \\
(10.40- \\
11.60) \\
214 / 3906 \\
(\% 5.5)\end{array}$} & \multirow[t]{3}{*}{$0.00^{\star \star \star *}$} \\
\hline & & & & $\begin{array}{l}231(\% \\
6.3)\end{array}$ & & $\begin{array}{l}6 / 214(\% \\
2.8)\end{array}$ & $\begin{array}{l}174 / 214 \\
(\% 81.3)\end{array}$ & $\begin{array}{l}34 / 214(\% \\
15.9)\end{array}$ & & \\
\hline & & 0.97 (fold) & & $\begin{array}{l}1.04 \\
\text { (fold) }\end{array}$ & & 0.97 (fold) & & $\begin{array}{l}1.08 \\
\text { (fold) }\end{array}$ & & \\
\hline \multirow[t]{3}{*}{ NEU (\%) } & \multirow[t]{3}{*}{$1.8-6.98$} & $\begin{array}{l}1.48 \\
(1.21- \\
1.62)\end{array}$ & $\begin{array}{l}3.62 \\
(2.75- \\
4.76)\end{array}$ & $\begin{array}{l}8.86 \\
(7.84- \\
10.61)\end{array}$ & \multirow{3}{*}{$\begin{array}{l}3.76(2.65- \\
5.51) \\
3745 / 3968 \\
(\% 94.4)\end{array}$} & $\begin{array}{l}1.35 \\
(0.64- \\
1.73)\end{array}$ & $\begin{array}{l}5.4 \\
(3.72- \\
6.30)\end{array}$ & $\begin{array}{l}11.24 \\
(8.98- \\
15.5)\end{array}$ & \multirow{3}{*}{$\begin{array}{l}8.58(5.95- \\
13.04) \\
223 / 3968 \\
(\% 5.6)\end{array}$} & \multirow[t]{3}{*}{$0.00^{* * *}$} \\
\hline & & $\begin{array}{l}274 / 3745 \\
(\% 7.3)\end{array}$ & $\begin{array}{l}2958 / 3745 \\
(\% 79.0)\end{array}$ & $\begin{array}{l}513 / 3745 \\
(\% 13.7)\end{array}$ & & $\begin{array}{l}5 / 223(\% \\
2.2)\end{array}$ & $\begin{array}{l}76 / 223 \\
(\% 34.1)\end{array}$ & $\begin{array}{l}142 / 223 \\
(\% 63.7)\end{array}$ & & \\
\hline & & 0.82 (fold) & & $\begin{array}{l}1.27 \\
\text { (fold) }\end{array}$ & & 0.75 (fold) & & $\begin{array}{l}1.61 \\
\text { (fold) }\end{array}$ & & \\
\hline \multirow[t]{3}{*}{ PCT (\%) } & \multirow[t]{3}{*}{$\begin{array}{l}0.18- \\
0.39\end{array}$} & $\begin{array}{l}0.15 \\
(0.13- \\
0.16)\end{array}$ & $\begin{array}{l}0.24 \\
(0.21- \\
0.29)\end{array}$ & $\begin{array}{l}0.43 \\
(0.41- \\
0.46)\end{array}$ & $\begin{array}{l}0.23(0.18- \\
0.28)\end{array}$ & $\begin{array}{l}0.15 \\
(0.13- \\
0.16)\end{array}$ & $\begin{array}{l}0.26 \\
(0.22- \\
0.31)\end{array}$ & $\begin{array}{l}0.43 \\
(0.41- \\
0.49)\end{array}$ & $\begin{array}{l}0.24(0.18- \\
0.31)\end{array}$ & $0.01^{*}$ \\
\hline & & $\begin{array}{l}801 / 3692 \\
(\% 21.7)\end{array}$ & $\begin{array}{l}2740 / 3692 \\
(\% 74.2)\end{array}$ & $\begin{array}{l}151 / 3692 \\
(4.1)\end{array}$ & $\begin{array}{l}3692 / 3906 \\
(\% 94.5)\end{array}$ & $\begin{array}{l}48 / 214(\% \\
22.4)\end{array}$ & $\begin{array}{l}147 / 214 \\
(\% \text { 68.7) }\end{array}$ & $\begin{array}{l}19 / 214(\% \\
8.9)\end{array}$ & $\begin{array}{l}214 / 3906 \\
(\% 5.5)\end{array}$ & \\
\hline & & 0.83 (fold) & & $\begin{array}{l}1.10 \\
\text { (fold) }\end{array}$ & & 0.83 (fold) & & $\begin{array}{l}1.10 \\
\text { (fold) }\end{array}$ & & \\
\hline PDW (fL) & $9.9-15.4$ & $\begin{array}{l}9.40(9.00- \\
9.70)\end{array}$ & $\begin{array}{l}11.9 \\
(10.9- \\
13.0)\end{array}$ & $\begin{array}{l}16.9 \\
(16.1- \\
18.3)\end{array}$ & $\begin{array}{l}11.7(10.5- \\
13.1)\end{array}$ & $\begin{array}{l}9.3(8.75- \\
9.6)\end{array}$ & $\begin{array}{l}12.65 \\
(11.65- \\
13.75)\end{array}$ & $\begin{array}{l}18.10 \\
(16.9- \\
19.5)\end{array}$ & $\begin{array}{l}12.75 \\
(11.60- \\
14.50)\end{array}$ & $0.00^{\star \star *}$ \\
\hline & & $\begin{array}{l}522 / 3692 \\
(\% 14.1)\end{array}$ & $\begin{array}{l}2943 / 3692 \\
(\% 79.7)\end{array}$ & $\begin{array}{l}227 / 3692 \\
(\% \text { 6.1) }\end{array}$ & $\begin{array}{l}3692 / 3906 \\
(\% 94.5)\end{array}$ & $\begin{array}{l}16 / 214(\% \\
7.5)\end{array}$ & $\begin{array}{l}168 / 214 \\
(\% 78.5)\end{array}$ & $\begin{array}{l}30 / 214(\% \\
14.0)\end{array}$ & $\begin{array}{l}214 / 3906 \\
(\% 5.5)\end{array}$ & \\
\hline & & 0.95 (fold) & & $\begin{array}{l}1.10 \\
\text { (fold) }\end{array}$ & & 0.94 (fold) & & $\begin{array}{l}1.18 \\
\text { (fold) }\end{array}$ & & \\
\hline
\end{tabular}

${ }^{*} \mathrm{p}<0.05,{ }^{* *} \mathrm{p}<0.01,{ }^{* * *} \mathrm{p}<0.001, \mathrm{p}$ values denoted comparison of total median values between ICU and non-ICU groups. BASO, basophil; d-NLR, derived neutrophil to lymphocyte ratio; EOS, eosinophil; HCT, hematocrit; HGB, hemoglobin; IQR, interquartile range; LMR, lymphocyte to monocyte ratio; LYM, lymphocyte; $\mathrm{MCH}$, mean corpuscular hemoglobin; $\mathrm{MCHC}$, mean corpuscular hemoglobin concentration; MCV, mean corpuscular volume; MONO, monocyte; MPV, mean platelet volume; NEU, neutrophil; NLR, neutrophil to lymphocyte ratio; PCT, plateletcrit; PDW, platelet distribution width; P-LRC, platelet large cell ratio; PLR, platelet to lymphocyte ratio; PLT, platelet; RBC, red blood cells; RDW, red cell distribution width; SD, standard deviation; WBC, white blood cell. 


\begin{tabular}{|c|c|c|c|c|c|c|c|c|c|c|}
\hline \multicolumn{6}{|l|}{ Non-ICU } & \multicolumn{5}{|l|}{ ICU } \\
\hline \multirow[t]{3}{*}{$\mathrm{PLT} \times 10^{\wedge} 3 / \mu \mathrm{L}$} & \multirow[t]{3}{*}{$150-450$} & $\begin{array}{l}127.0 \\
(108.0- \\
140.0)\end{array}$ & $\begin{array}{l}230.0 \\
(192.0- \\
279.0)\end{array}$ & $\begin{array}{l}492.0 \\
(468.0- \\
533.0)\end{array}$ & $\begin{array}{l}218.0 \\
(173.0- \\
272.0)\end{array}$ & $\begin{array}{l}119.0 \\
(95.0- \\
135.0)\end{array}$ & $\begin{array}{l}244.0 \\
(195.0- \\
309.0)\end{array}$ & $\begin{array}{l}480.0 \\
(456.0- \\
521.5)\end{array}$ & $\begin{array}{l}220.0 \\
(153.0- \\
296.0)\end{array}$ & \multirow[t]{3}{*}{0.93} \\
\hline & & $\begin{array}{l}549 / 3744 \\
(\% 14.7)\end{array}$ & $\begin{array}{l}3132 / 3744 \\
(\% 83.7)\end{array}$ & $\begin{array}{l}63 / 3744 \\
(\% 1.7)\end{array}$ & $\begin{array}{l}3744 / 3967 \\
(\% 94.4)\end{array}$ & $\begin{array}{l}46 / 223(\% \\
20.6)\end{array}$ & $\begin{array}{l}173 / 223 \\
(\% 77.6)\end{array}$ & $\begin{array}{l}4 / 223(\% \\
1.8)\end{array}$ & $\begin{array}{l}223 / 3967 \\
(\% 5.6)\end{array}$ & \\
\hline & & \multicolumn{2}{|l|}{0.85 (fold) } & \multicolumn{2}{|l|}{$\begin{array}{l}1.09 \\
\text { (fold) }\end{array}$} & \multicolumn{2}{|l|}{0.79 (fold) } & \multicolumn{2}{|l|}{$\begin{array}{l}1.07 \\
\text { (fold) }\end{array}$} & \\
\hline \multirow[t]{3}{*}{$\mathrm{RBC} \times 10^{\wedge} 6 / \mu \mathrm{L}$} & \multirow[t]{3}{*}{$4.5-6$} & $\begin{array}{l}4.17 \\
(3.91- \\
4.36)\end{array}$ & $\begin{array}{l}4.96 \\
(4.72- \\
5.28)\end{array}$ & $\begin{array}{l}6.16 \\
(6.07- \\
6.36)\end{array}$ & \multirow{3}{*}{$\begin{array}{l}4.7(4.32- \\
5.10) \\
3745 / 3968 \\
(\% 94.4)\end{array}$} & \multirow{2}{*}{$\begin{array}{l}3.93 \\
(3.40- \\
4.20) \\
129 / 223 \\
(\% 57.8)\end{array}$} & $\begin{array}{l}4.86 \\
(4.72- \\
5.20)\end{array}$ & $\begin{array}{l}6.18 \\
(6.14- \\
6.33)\end{array}$ & $\begin{array}{l}4.30(3.79- \\
4.82)\end{array}$ & \multirow[t]{3}{*}{$0.00^{\star \star \star *}$} \\
\hline & & $\begin{array}{l}1341 / 3745 \\
(\% 35.8)\end{array}$ & $\begin{array}{l}2344 / 3745 \\
(\% \text { 62.6) }\end{array}$ & $\begin{array}{l}60 / 3745 \\
(\% 1.6)\end{array}$ & & & $\begin{array}{l}88 / 223 \\
(\% 39.5)\end{array}$ & $\begin{array}{l}6 / 223(\% \\
2.7)\end{array}$ & $\begin{array}{l}223 / 3968 \\
(\% 5.6)\end{array}$ & \\
\hline & & \multicolumn{2}{|l|}{0.93 (fold) } & $\begin{array}{l}1.03 \\
\text { (fold) }\end{array}$ & & \multicolumn{2}{|l|}{0.87 (fold) } & \multicolumn{2}{|l|}{$\begin{array}{l}1.03 \\
\text { (fold) }\end{array}$} & \\
\hline \multirow[t]{3}{*}{ RDW (\%) } & \multirow[t]{3}{*}{$11-14$} & & $\begin{array}{l}12.80 \\
(12.3- \\
13.2)\end{array}$ & $\begin{array}{l}15.10 \\
(14.5- \\
16.4)\end{array}$ & \multirow{3}{*}{$\begin{array}{l}13.1(12.5- \\
14 .) \\
3743 / 3966 \\
(\% 94.4)\end{array}$} & \multirow[t]{3}{*}{-} & $\begin{array}{l}13.30 \\
(12.9- \\
13.7)\end{array}$ & $\begin{array}{l}15.6 \\
(14.6- \\
18.30)\end{array}$ & $\begin{array}{l}14.40 \\
(13.40- \\
16.10)\end{array}$ & \multirow[t]{3}{*}{$0.00^{\text {*kx }}$} \\
\hline & & & $\begin{array}{l}2836 / 3743 \\
(\% 75.8)\end{array}$ & $\begin{array}{l}906 / 3743 \\
(\% 24.2)\end{array}$ & & & $\begin{array}{l}97 / 223 \\
(\% 43.5)\end{array}$ & $\begin{array}{l}126 / 223 \\
(\% 56.5)\end{array}$ & $\begin{array}{l}223 / 3966 \\
(\% 5.6)\end{array}$ & \\
\hline & & & & $\begin{array}{l}1.08 \\
\text { (fold) }\end{array}$ & & & & $\begin{array}{l}1.11 \\
\text { (fold) }\end{array}$ & & \\
\hline \multirow[t]{6}{*}{$W B C \times 10^{\wedge} 3 / \mu \mathrm{L}$} & \multirow{3}{*}{$\begin{array}{l}\text { Female } \\
4.49- \\
12.68\end{array}$} & $\begin{array}{l}3.7(3.1- \\
4.1)\end{array}$ & $\begin{array}{l}6.4(5.4- \\
7.8)\end{array}$ & $\begin{array}{l}13.7 \\
(13.2- \\
15.9)\end{array}$ & \multirow{3}{*}{$\begin{array}{l}5.70(4.5- \\
7.45) \\
1856 / 1944 \\
(\% 95.5)\end{array}$} & \multirow{3}{*}{$\begin{array}{l}3.15(2.3- \\
3.8) \\
10 / 88(\% \\
11.4) \\
0.70 \text { (fold) }\end{array}$} & \multirow{3}{*}{$\begin{array}{l}8.0(6.6- \\
9.5) \\
49 / 88(\% \\
55.7)\end{array}$} & \multirow{3}{*}{$\begin{array}{l}17.5 \\
(15.5- \\
21.3) \\
29 / 88(\% \\
33.0) \\
1.38 \\
\text { (fold) }\end{array}$} & \multirow{3}{*}{$\begin{array}{l}9.3(6.5- \\
15.35) \\
88 / 1944(\% \\
4.5)\end{array}$} & \multirow[t]{3}{*}{$0.00^{\text {*** }}$} \\
\hline & & $\begin{array}{l}458 / 1856 \\
(\% 24.7)\end{array}$ & $\begin{array}{l}1363 / 1856 \\
(\% 73.4)\end{array}$ & $\begin{array}{l}15.9) \\
35 / 1856 \\
(\% 1.9)\end{array}$ & & & & & & \\
\hline & & 0.83 (fold) & & $\begin{array}{l}1.08 \\
\text { (fold) }\end{array}$ & & & & & & \\
\hline & Male & $\begin{array}{l}3.4(3.0- \\
3.7)\end{array}$ & $\begin{array}{l}6.6(5.4- \\
7.9)\end{array}$ & $\begin{array}{l}13.1 \\
(11.9-\end{array}$ & $\begin{array}{l}6.6(5.1- \\
8.45)\end{array}$ & $\begin{array}{l}3.3(2.6- \\
3.4)\end{array}$ & $\begin{array}{l}8.6(7.2- \\
9.6)\end{array}$ & $\begin{array}{l}15.75 \\
(12.8-\end{array}$ & $\begin{array}{l}10.8(8.2- \\
15.60)\end{array}$ & $0.00^{\star \star *}$ \\
\hline & 10.9 & $\begin{array}{l}163 / 1868 \\
(\% 8.7)\end{array}$ & $\begin{array}{l}1518 / 1868 \\
(\% 81.3)\end{array}$ & $\begin{array}{l}187 / 1868 \\
(\% \text { 10.0) }\end{array}$ & $\begin{array}{l}1868 / 2002 \\
(\% 93.3)\end{array}$ & $\begin{array}{l}5 / 134(\% \\
3.7)\end{array}$ & $\begin{array}{l}65 / 134 \\
(\% 48.5)\end{array}$ & $\begin{array}{l}64 / 134(\% \\
47.8)\end{array}$ & $\begin{array}{l}134 / 2002 \\
(\% 6.7)\end{array}$ & \\
\hline & & 0.87 (fold) & & 1.2 (fold) & & 0.84 (fold) & & $\begin{array}{l}1.44 \\
\text { (fold) }\end{array}$ & & \\
\hline P.LCR (\%) & & & & & $\begin{array}{l}27.8(23.2- \\
33.1)\end{array}$ & & & & $\begin{array}{l}32.20 \\
(27.40- \\
37.50)\end{array}$ & $0.00^{\star \star \star}$ \\
\hline NLR & & & & & $\begin{array}{l}2.40(1.54- \\
4.50)\end{array}$ & & & & $\begin{array}{l}12.10 \\
(5.76- \\
19.85)\end{array}$ & $0.00^{\star \star *}$ \\
\hline PLR & & & & & $\begin{array}{l}142.55 \\
(104.82- \\
218.43)\end{array}$ & & & & $\begin{array}{l}293.48 \\
(169.49- \\
468.09)\end{array}$ & $0.00^{\star \star *}$ \\
\hline dNLR & & & & & $\begin{array}{l}1.68(1.15- \\
2.90)\end{array}$ & & & & $\begin{array}{l}6.56(3.39- \\
10.70)\end{array}$ & $0.00^{* \star *}$ \\
\hline LMR & & & & & $\begin{array}{l}3.13(2.07- \\
4.29)\end{array}$ & & & & $\begin{array}{l}1.85(1.10- \\
2.91)\end{array}$ & $0.00^{\star \star *}$ \\
\hline $\begin{array}{l}{ }^{*} \mathrm{p}<0.05,{ }^{* *} \mathrm{p}<0 . \\
\text { neutrophil to lyn } \\
\text { lymphocyte; MC } \\
\text { MPV, mean plat } \\
\text { ratio; PLR, plate }\end{array}$ & $\begin{array}{l}\text { ean corp } \\
\text { olume; } \\
\text { lymphoc }\end{array}$ & $\begin{array}{l}\text { values deno } \\
\text { S, eosinoph } \\
\text { ular hemogl } \\
\text { neutrophil; } \\
\text { ratio; PLT, pl }\end{array}$ & $\begin{array}{l}\text { d comparisc } \\
\text { HCT, hemat } \\
\text { in; MCHC, m } \\
\text { R, neutrophi } \\
\text { elet; } R B C \text {, re }\end{array}$ & $\begin{array}{l}\text { of total me } \\
\text { it; HGB, he } \\
\text { n corpusc } \\
\text { lymphocy } \\
\text { lood cells; }\end{array}$ & $\begin{array}{l}\text { n values betv } \\
\text { globin; IQR, i } \\
\text { hemoglobin } \\
\text { atio; PCT, pla } \\
\text { W, red cell dis }\end{array}$ & $\begin{array}{l}\text { en ICU and n } \\
\text { rquartile ran } \\
\text { ncentration; } \\
\text { etcrit; PDW, } \\
\text { bution width }\end{array}$ & $\begin{array}{l}\text { n-ICU gro } \\
\text { ye; LMR, ly } \\
\text { MCV, mear } \\
\text { latelet dis } \\
\text { SD, stand }\end{array}$ & $\begin{array}{l}\text { BASO, bas } \\
\text { locyte to } n \\
\text { puscular v } \\
\text { tion width } \\
\text { deviation; }\end{array}$ & $\begin{array}{l}\text { hil; d-NLR, deri } \\
\text { ocyte ratio; LY } \\
\text { Ime; MONO, m } \\
\text { LRC, platelet l } \\
\text { C, white blood }\end{array}$ & $\begin{array}{l}\text { ed } \\
\text { h, } \\
\text { nocyte; } \\
\text { gee cell } \\
\text { cell. }\end{array}$ \\
\hline
\end{tabular}

In the non-ICU group, alanine aminotransferase (ALT) and gamma-glutamyltransferase (GGT) values gradually increased from spring to autumn while albumin, alkaline phosphatase (ALP), calcium, total bilirubin, total protein, and estimated glomerular filtration rate (eGFR) gradually decreased. Additionally, aspartate aminotransferase (AST) was lower in spring than other seasons and HDL was higher in spring than other seasons while AST/ALT, direct bilirubin and chlorine were lower in autumn than other seasons. Moreover, glucose, creatinine (only in men), urea and uric acid were higher in autumn than in other seasons, and lactate dehydrogenase (LDH) was higher in summer than in other seasons while creatine kinase myocardial band (CK-MB), creatinine (only in women) and lipase were lower in summer than autumn and cholesterol were higher in spring than autumn. Also, sodium was higher in summer than autumn and AIP was higher in summer than spring. In the ICU group, ALT, GGT and creatinine (only in men) increased in summer than autumn, and AST, glucose and 
urea were higher in autumn than other seasons while calcium and eGFR were lower in autumn than other seasons and, albumin and ALP were higher in spring than other seasons. Additionally, LDH was higher in autumn than spring and the total protein value was lower in autumn than spring (Table 3 ). 
Seasonal comparison of biochemical biomarkers in the in Non-ICU

\section{Spring}

\begin{tabular}{|c|c|c|c|c|c|c|c|c|c|c|c|c|c|c|}
\hline Test & Mean & Med & Min & Max & SD & Mean & Med & Min & Max & SD & Mean & Med & Min & Max \\
\hline $\operatorname{ALT}(\mathrm{U} / \mathrm{L})$ & $29.6^{\#}$ & 18.0 & 4.0 & 910.0 & 51.13 & $33.75^{\star}$ & 22.0 & 3.0 & 1349.0 & 54.52 & $35.51^{\llbracket}$ & 24.85 & .70 & 960.0 \\
\hline AST (U/L) & $30.54^{\rrbracket}$ & 23.0 & 9.0 & 1062.0 & 55.85 & $34.12^{*}$ & 27.0 & 9.0 & 1062.0 & 41.36 & $33.67^{*}$ & 26.0 & 4.0 & 674.0 \\
\hline AST/ALT & $1.31^{\#}$ & 1.17 & 0.22 & 5.5 & 0.69 & $1.32^{\#}$ & 1.2 & 0.22 & 6.33 & 0.68 & $1.24^{\star}$ & 1.05 & 0.15 & 102.8 \\
\hline Alb (g/L) & $39.90^{\#}$ & 40.30 & 17.70 & 49.79 & 5.26 & $38.76^{*}$ & 39.00 & 0.08 & 55.0 & 5.42 & $37.29^{\rrbracket}$ & 36.9 & 0.17 & 52.1 \\
\hline ALP (U/L) & $441.25^{\#}$ & 95.0 & 5.60 & 3150.0 & 497.45 & $189.32^{*}$ & 77.0 & 1.0 & 3150.0 & 330.55 & $73.42^{\llbracket}$ & 72.00 & 3.60 & 464.0 \\
\hline $\begin{array}{l}\text { Amylase } \\
(\mathrm{U} / \mathrm{L})\end{array}$ & 69.77 & 64.0 & 24.0 & 174.00 & 29.51 & 72.90 & 65.00 & 11.0 & 266.0 & 35.29 & 76.78 & 67.00 & 16.00 & 874.0 \\
\hline $\begin{array}{l}\text { CK-MB } \\
(\mathrm{U} / \mathrm{L})\end{array}$ & $18.84^{\star \rrbracket /}$ & 16.10 & 6.30 & 174.70 & 13.33 & $19.93^{*}$ & 16.7 & 4.80 & 575.4 & 23.26 & $18.15^{\rrbracket}$ & 15.20 & 1.00 & 211.4 \\
\hline $\begin{array}{l}\text { Iron } \\
(\mu \mathrm{g} / \mathrm{dL})\end{array}$ & 51.1 & 50.0 & 1.0 & 220.00 & 37.1 & 47.2 & 45.0 & 1.0 & 220.0 & 35.3 & 33.80 & 23.00 & 10.00 & 61.00 \\
\hline $\begin{array}{l}\text { TIBC } \\
(\mu \mathrm{g} / \mathrm{dL})\end{array}$ & 243.9 & 237.0 & 48.0 & 379.00 & 70.4 & 247.6 & 238.0 & 48.0 & 379.0 & 67.3 & 237.75 & 222.00 & 161.00 & 346.0 \\
\hline $\begin{array}{l}\text { D. Bil. } \\
\text { (mg/dL) }\end{array}$ & $0.15^{\#}$ & 0.13 & 0.03 & 1.33 & 0.11 & $0.18^{\#}$ & 0.12 & 0.01 & 20.0 & 0.90 & $0.14^{\star}$ & 0.10 & 0.01 & 13.00 \\
\hline GGT (U/L) & $33.9^{\#}$ & 24.0 & 7.0 & 282.00 & 30.61 & $45.55^{\star}$ & 28.0 & 1.0 & 1085.0 & 61.32 & $50.89^{\rrbracket}$ & 32.00 & 1.00 & 664.0 \\
\hline $\begin{array}{l}\text { Glucose } \\
\text { (mg/dL) }\end{array}$ & $107.97^{\neq}$ & 96.0 & 31.0 & 434.00 & 43.1 & $113.0^{\neq}$ & 98.0 & 17.0 & 527.0 & 51.0 & $148.54^{\star}$ & 118.0 & 36.00 & 695.0 \\
\hline $\begin{array}{l}\mathrm{HDL} \\
(\mathrm{mg} / \mathrm{dL})\end{array}$ & $38.3^{*}$ & 36.0 & 13.0 & 76.00 & 11.3 & $34.55^{\#}$ & 33.0 & 13.0 & 93.0 & 10.04 & $33.64^{\#}$ & 32.00 & 15.00 & 71.00 \\
\hline $\begin{array}{l}\mathrm{Ca} \\
(\mathrm{mg} / \mathrm{dL})\end{array}$ & $9.34^{\#}$ & 9.4 & 6.8 & 11.70 & 0.60 & $9.0^{*}$ & 9.0 & 0.6 & 11.7 & 0.7 & $8.80^{\rrbracket}$ & 8.73 & 6.70 & 12.43 \\
\hline $\begin{array}{l}\mathrm{Cl} \\
(\mathrm{mmol} / \mathrm{L})\end{array}$ & $103.64^{\neq}$ & 104.0 & 90.00 & 119.00 & 3.62 & $104.0^{\neq}$ & 104.0 & 90.0 & 125.0 & 3.75 & $102.91^{*}$ & 103.00 & 84.00 & 126.0 \\
\hline $\begin{array}{l}\text { T.Col. } \\
\text { (mg/dL) }\end{array}$ & $171.05^{\star}$ & 164.00 & 73.00 & 338.00 & 46.78 & $161.54^{\text {枢 }}$ & 158.0 & 6.0 & 338.0 & 40.61 & $160.67^{\rrbracket}$ & 158.00 & 5.00 & 354.0 \\
\hline Male & $1.08^{\neq}$ & 0.99 & 0.54 & 4.78 & 0.43 & $1.09^{\neq}$ & 0.99 & 0.54 & 6.8 & 0.5 & $1.32^{*}$ & 1.03 & 0.55 & 202.0 \\
\hline $\begin{array}{l}\text { Creatinine. } \\
(\mathrm{mg} / \mathrm{dL})\end{array}$ & $0.87^{\neq^{*}}$ & 0.80 & 0.54 & 2.56 & 0.28 & $0.88^{\neq}$ & 0.81 & 0.46 & 6.34 & 0.37 & $0.92^{*}$ & 0.83 & 0.32 & 8.84 \\
\hline
\end{tabular}

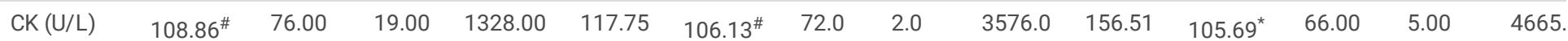

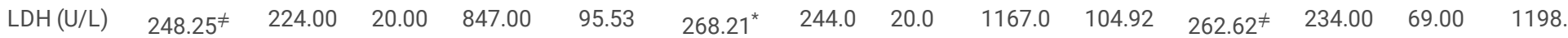

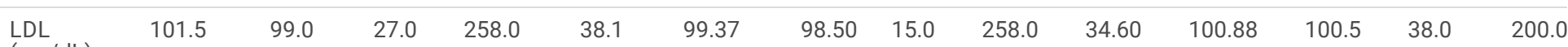

$(\mathrm{mg} / \mathrm{dL})$

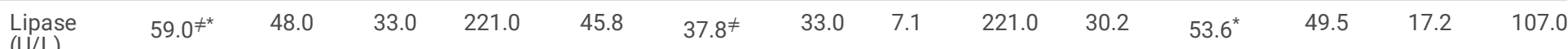

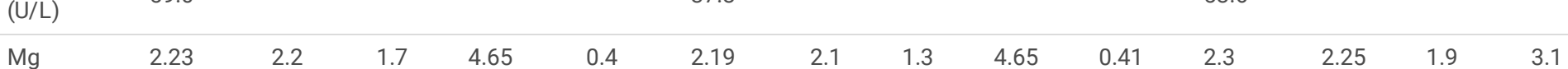

$(\mathrm{mg} / \mathrm{dL})$

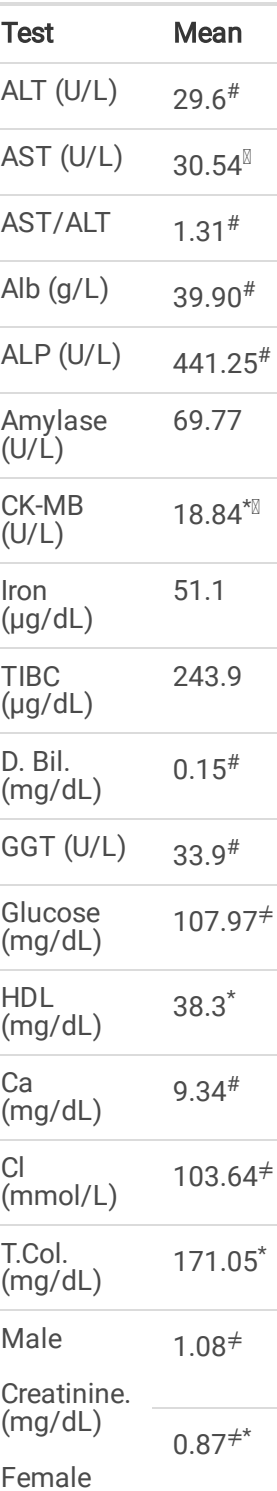

$(\mathrm{mmol} / \mathrm{L})$

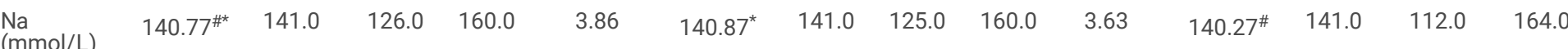

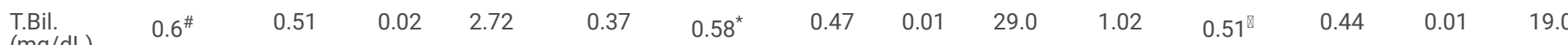

$(\mathrm{mg} / \mathrm{dL})$

Summer

Autumn 


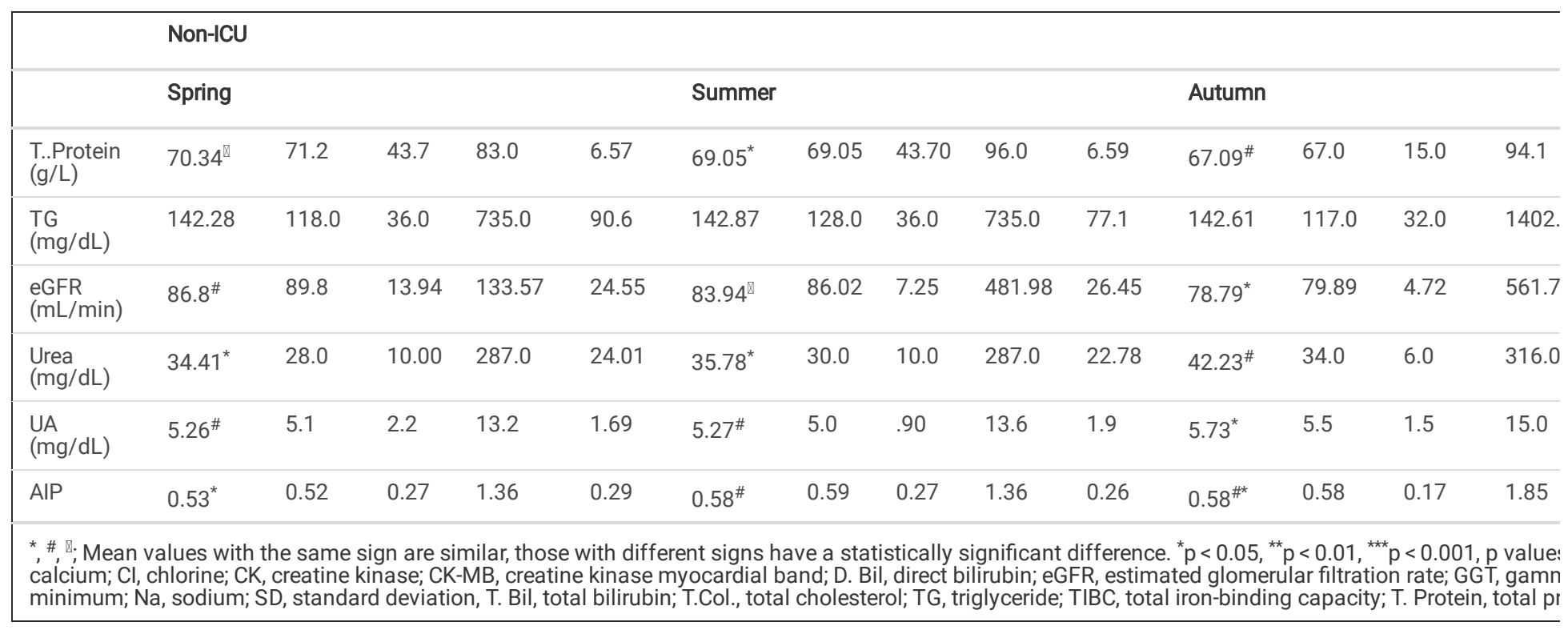

While ALT, AST, AST/ALT, ALP, CK-MB, direct bilirubin, GGT, glucose, creatinine, creatine kinase (CK), LDH, magnesium, sodium, total bilirubin and urea were higher, albumin, iron, calcium, total protein and eGFR were lower in the ICU group compared to the non-ICU group (Table 4). 
Table 4

Comparison of biochemical biomarkers in the intensive care unit (ICU) and non-ICU groups with coronavirus disease 2019 on admission.

\begin{tabular}{|c|c|c|c|c|c|c|c|c|c|c|}
\hline \multicolumn{3}{|l|}{ Non-ICU } & \multicolumn{8}{|c|}{ ICU } \\
\hline \multirow[t]{3}{*}{ Test } & \multirow{3}{*}{$\begin{array}{l}\text { Reference } \\
\text { range }\end{array}$} & Decreased & Normal & Increased & Total & Decreased & Normal & Increased & Total & $\mathbf{p}$ \\
\hline & & \multirow{2}{*}{$\begin{array}{l}\text { Median } \\
\text { (IQR) } n / n \\
(\%)\end{array}$} & $\begin{array}{l}\text { Median } \\
\text { (IQR) }\end{array}$ & $\begin{array}{l}\text { Median } \\
\text { (IQR) }\end{array}$ & $\begin{array}{l}\text { Median } \\
\text { (IQR) }\end{array}$ & \multirow{2}{*}{$\begin{array}{l}\text { Median } \\
\text { (IQR) } n / n \\
(\%)\end{array}$} & \multirow{2}{*}{$\begin{array}{l}\text { Median } \\
\text { (IQR) n/n } \\
(\%)\end{array}$} & $\begin{array}{l}\text { Median } \\
\text { (IQR) }\end{array}$ & \multirow{2}{*}{$\begin{array}{l}\text { Median } \\
\text { (IQR) n/n } \\
(\%)\end{array}$} & \\
\hline & & & $\mathrm{n} / \mathrm{n}(\%)$ & $\mathrm{n} / \mathrm{n}(\%)$ & $\mathrm{n} / \mathrm{n}(\%)$ & & & $\mathrm{n} / \mathrm{n}(\%)$ & & \\
\hline \multirow{4}{*}{$\begin{array}{l}\text { ALT } \\
(\mathrm{U} / \mathrm{L})\end{array}$} & \multirow[t]{4}{*}{$0-35$} & \multirow[t]{4}{*}{-} & \multirow{2}{*}{$\begin{array}{l}18.0 \\
(14.0- \\
25.0)\end{array}$} & $\begin{array}{l}54.0(43.0- \\
74.10)\end{array}$ & $\begin{array}{l}23.0(16.0- \\
39.0)\end{array}$ & & \multirow{2}{*}{$\begin{array}{l}19.0 \\
(14.0- \\
26.0)\end{array}$} & $\begin{array}{l}63.0(46.0- \\
102.6)\end{array}$ & $\begin{array}{l}32.0(19.0- \\
61.0)\end{array}$ & \multirow[t]{4}{*}{$0.00^{\text {** }}$} \\
\hline & & & & $1116 / 3944$ & $3944 / 4183$ & & & $112 / 239 \%$ & 239/4183(\% & \\
\hline & & & \multirow{2}{*}{$\begin{array}{l}2828 / 3944 \\
(\% 71.7)\end{array}$} & $(\% 28.3)$ & $(\% 94.3)$ & & \multirow{2}{*}{$\begin{array}{l}127 / 239 \\
(\% 53.1)\end{array}$} & 46.9) & $5.7)$ & \\
\hline & & & & 1.54 (fold) & & & & 1.8 (fold) & & \\
\hline \multirow{4}{*}{$\begin{array}{l}\text { AST } \\
(\mathrm{U} / \mathrm{L})\end{array}$} & \multirow[t]{4}{*}{$0-50$} & \multirow[t]{4}{*}{-} & \multirow{2}{*}{$\begin{array}{l}24.0 \\
(19.0- \\
32.0)\end{array}$} & $\begin{array}{l}66.0(56.0- \\
86.5)\end{array}$ & $\begin{array}{l}26.0(20.0- \\
37.0)\end{array}$ & & $\begin{array}{l}28.0 \\
(21.5- \\
36.5)\end{array}$ & $\begin{array}{l}79.5(60.0- \\
150.5)\end{array}$ & $\begin{array}{l}44.0(26.0- \\
70.0)\end{array}$ & \multirow[t]{4}{*}{$0.00^{\text {** }}$} \\
\hline & & & & $464 / 3864$ & $3864 / 4104$ & & & $100 / 240(\%$ & $240 / 4104$ & \\
\hline & & & \multirow{2}{*}{$\begin{array}{l}3400 / 3864 \\
(\% 88.0)\end{array}$} & (\% 12.0) & $(\%$ 94.2) & & $\begin{array}{l}140 / 240 \\
(\% 58.3)\end{array}$ & 41.7) & (\% 5.8) & \\
\hline & & & & 1.32 (fold) & & & & 1.59 (fold) & & \\
\hline AST/ALT & & & & & $\begin{array}{l}1.11(0.81- \\
1.50)\end{array}$ & & & & $\begin{array}{l}1.33(0.88- \\
2.05)\end{array}$ & $0.00^{* *}$ \\
\hline $\begin{array}{l}\text { Alb } \\
(g / L)\end{array}$ & $35-52$ & $\begin{array}{l}32.4(30.5- \\
33.7)\end{array}$ & $\begin{array}{l}40.0 \\
(37.4- \\
43.3)\end{array}$ & $\begin{array}{l}52.94 \\
(52.19- \\
54.31)\end{array}$ & $\begin{array}{l}37.90 \\
(34.30- \\
4200)\end{array}$ & $\begin{array}{l}27.8(24.1- \\
30.8)\end{array}$ & $\begin{array}{l}37.5(36.0- \\
41.4)\end{array}$ & & $\begin{array}{l}29.25 \\
(25.4-32.6)\end{array}$ & $0.00^{\star *}$ \\
\hline & & $\begin{array}{l}893 / 3108 \\
(\% 28.7)\end{array}$ & $2211 / 3108$ & 4/3108 (\% & $3108 / 3324$ & $\begin{array}{l}179 / 216(\% \\
82.9)\end{array}$ & $\begin{array}{l}37 / 216(\% \\
17.1)\end{array}$ & & $\begin{array}{l}216 / 3324 \\
(\% 6.5)\end{array}$ & \\
\hline & & 0.92 (fold) & & & & 0.74 (fold) & & & & \\
\hline & & & & 1.02 (fold) & & & & & & \\
\hline ALP & $30-120$ & $\begin{array}{l}7.70(6.30- \\
9.4)\end{array}$ & $\begin{array}{l}73.0 \\
(60.0-\end{array}$ & $\begin{array}{l}580.0 \\
(140.5-\end{array}$ & $\begin{array}{l}75.0(56.0- \\
100.0)\end{array}$ & $\begin{array}{l}7.35(6.8- \\
7.9)\end{array}$ & $\begin{array}{l}75.5 \\
(63.5-\end{array}$ & $\begin{array}{l}266.0 \\
(140.0-\end{array}$ & $\begin{array}{l}87.0(66.0- \\
128.0)\end{array}$ & $0.00^{* *}$ \\
\hline$(\mathrm{U} / \mathrm{L})$ & & $556 / 2541$ & & 900.0) & $2541 / 2732$ & 20/191 & & & $191 / 1732$ & \\
\hline & & (\% 21.9) & $\begin{array}{l}1597 / 2541 \\
(\% \text { 62.8) }\end{array}$ & $\begin{array}{l}388 / 2541 \\
(\% 15.3)\end{array}$ & $(\%$ 93.0) & (\%10.5) & $\begin{array}{l}124 / 191 \\
(\% 64.9)\end{array}$ & $\begin{array}{l}4 / / 191(\% \\
24.6)\end{array}$ & $(\% /)$ & \\
\hline & & $0 . \angle 0$ (ioid) & & 4.83 (fold) & & 0.24 & & 2.22 (fold) & & \\
\hline $\begin{array}{l}\text { Amylase } \\
(\mathrm{U} / \mathrm{L})\end{array}$ & $28-100$ & $\begin{array}{l}24.0(20.0- \\
25.0)\end{array}$ & $\begin{array}{l}62.0 \\
(50.0- \\
77.0)\end{array}$ & $\begin{array}{l}120.0 \\
(106.5- \\
143.5)\end{array}$ & $\begin{array}{l}65.0(51.0- \\
88.0)\end{array}$ & $\begin{array}{l}21.0(18.0- \\
26.0)\end{array}$ & $\begin{array}{l}52.0 \\
(42.0-\end{array}$ & $\begin{array}{l}169.0 \\
(137.0-\end{array}$ & $\begin{array}{l}64.0(44.0- \\
105.0)\end{array}$ & 0.71 \\
\hline & & 14/708 (\% & $1 / 708$ & 108 & 708/793 (\% & $7 / 85$ (\% & & & $85 / 793(\%$ & \\
\hline & & & (\% 82.8) & 15.3) & & & 64.7) & 27.1) & & \\
\hline & & 0.00 (1010) & & 1.2 (fold) & & & & 1.69 (fold) & & \\
\hline $\begin{array}{l}\text { CK-MB } \\
(\mathrm{U} / \mathrm{L})\end{array}$ & $0-24$ & & $\begin{array}{l}14.9 \\
(11.9- \\
18.2)\end{array}$ & $\begin{array}{l}30.1(26.6- \\
44.25)\end{array}$ & $\begin{array}{l}16.00 \\
(12.5- \\
21.3)\end{array}$ & & $\begin{array}{l}16.9 \\
(14.7- \\
20.6)\end{array}$ & $\begin{array}{l}35.0(28.5- \\
46.3)\end{array}$ & $\begin{array}{l}25.35(17.1- \\
35.95)\end{array}$ & $0.00^{* *}$ \\
\hline & & & $1434 / 1706$ & $\begin{array}{l}272 / 1706 \\
(\% 15.9)\end{array}$ & $1706 / 1794$ & & $41 / 88(\%$ & $\begin{array}{l}47 / 88(\% \\
53.4)\end{array}$ & $\begin{array}{l}88 / 1794(\% \\
4.9)\end{array}$ & \\
\hline & & & & 1.25 (fold) & & & & 1.46 (fold) & & \\
\hline Iron & $70-180$ & $\begin{array}{l}42.0(21.0- \\
54.0)\end{array}$ & $\begin{array}{l}89.0 \\
(77.0-\end{array}$ & $\begin{array}{l}220.0 \\
(220.0-\end{array}$ & $\begin{array}{l}46.0(24.0- \\
62.0)\end{array}$ & $\begin{array}{l}11.50(5.0- \\
39.0)\end{array}$ & & & $\begin{array}{l}11.5(5.0- \\
39.0)\end{array}$ & $0.00^{*}$ \\
\hline & & $82 / 98$ & & 0 & $98 / 104$ & $6 / 6$ & & & $6 / 104$ & \\
\hline & & & $\begin{array}{l}14 / 98 \\
(\% 14.3)\end{array}$ & $2)$ & & 0) & & & .8) & \\
\hline & & 0.6 (fold) & & 1.22 (fold) & & 0.16 (fold) & & & & \\
\hline $\begin{array}{l}\text { TIBC } \\
(\mu \mathrm{g} / \mathrm{dL})\end{array}$ & $155-355$ & $\begin{array}{l}146.0 \\
(48.0- \\
153.0)\end{array}$ & $\begin{array}{l}237.0 \\
(201.0- \\
279.0)\end{array}$ & $\begin{array}{l}377.0 \\
(376.0- \\
379.0)\end{array}$ & $\begin{array}{l}237.0 \\
(194.0- \\
297.0)\end{array}$ & $\begin{array}{l}124.0 \\
(124.0- \\
124.0)\end{array}$ & $\begin{array}{l}307.0 \\
(271.0- \\
307.0)\end{array}$ & & $\begin{array}{l}289.0 \\
(197.5- \\
307.0)\end{array}$ & 0.75 \\
\hline & & $\begin{array}{l}6 / 90(\% \\
6.7)\end{array}$ & $\begin{array}{l}78 / 90(\% \\
86.7)\end{array}$ & $\begin{array}{l}6 / 90(\% \\
6.7)\end{array}$ & $\begin{array}{l}90 / 94(\% \\
95.7)\end{array}$ & $\begin{array}{l}1 / 4(\% \\
25.0)\end{array}$ & $\begin{array}{l}3 / 4(\% \\
75.0)\end{array}$ & & $4 / 94(\% 4.3)$ & \\
\hline & & 0.94 (fold) & & 1.06 (fold) & & 0.8 (fold) & & & & \\
\hline
\end{tabular}

${ }^{*} \mathrm{p}<0.05,{ }^{* *} \mathrm{p}<0.01,{ }^{* *} \mathrm{p}<0.001$, p values denoted comparison of total median values between ICU and non-ICU groups. AIP, atherogenic index of plasma; Alb, albumin; ALP, alkaline phosphatase; ALT, alanine aminotransferase; AST, aspartate aminotransferase; Ca, calcium; Cl, chlorine; CK, creatine kinase; CK$\mathrm{MB}$, creatine kinase myocardial band; D. Bil, direct bilirubin; eGFR, estimated glomerular filtration rate; GGT, gamma glutamyltransferase; HDL, high density lipoprotein; IQR, interquartile range; K, potassium; LDH, lactate dehydrogenase; LDL, low density lipoprotein; Mg, magnesium; Na, sodium; T. Bil, total bilirubin; T.Col., total cholesterol; TG, triglyceride; TIBC, total iron binding capacity; T. Protein, total protein, UA, uric acid. 


\begin{tabular}{|c|c|c|c|c|c|c|c|c|c|c|}
\hline \multicolumn{3}{|l|}{ Non-ICU } & \multicolumn{8}{|c|}{ ICU } \\
\hline \multirow[t]{3}{*}{$\begin{array}{l}\text { D. Bil. } \\
\text { (mg/dL) }\end{array}$} & \multirow{3}{*}{\multicolumn{2}{|c|}{$0-0.2$}} & $\begin{array}{l}0.11 \\
(0.08- \\
0.14)\end{array}$ & $\begin{array}{l}0.26(0.22- \\
0.32)\end{array}$ & \multicolumn{2}{|l|}{$\begin{array}{l}0.11(0.08- \\
0.15)\end{array}$} & $\begin{array}{l}0.12 \\
(0.10- \\
0.14)\end{array}$ & $\begin{array}{l}0.28(0.22- \\
0.35)\end{array}$ & $\begin{array}{l}0.17(0.12- \\
0.26)\end{array}$ & \multirow[t]{3}{*}{$0.00^{* *}$} \\
\hline & & & \multirow{2}{*}{$\begin{array}{l}2484 / 2770 \\
(\% 89.7)\end{array}$} & $\begin{array}{l}286 / 2770 \\
(\% 10.3)\end{array}$ & \multirow[t]{2}{*}{$\begin{array}{l}2770 / 2963 \\
(\% 93.5)\end{array}$} & & \multirow{2}{*}{$\begin{array}{l}108 / 193 \\
(\% 56.0)\end{array}$} & $\begin{array}{l}85 / 193(\% \\
44.0)\end{array}$ & \multirow[t]{2}{*}{$\begin{array}{l}193 / 2963 \\
(\% 6.5)\end{array}$} & \\
\hline & & & & 1.3 (fold) & & & & 1.4 (fold) & & \\
\hline \multirow[t]{3}{*}{$\begin{array}{l}\text { GGT } \\
(\mathrm{U} / \mathrm{L})\end{array}$} & \multirow[t]{3}{*}{$0-38$} & & $\begin{array}{l}21.0 \\
(15.0- \\
28.0)\end{array}$ & $\begin{array}{l}64.0(48.0- \\
97.0)\end{array}$ & \multirow{3}{*}{$\begin{array}{l}30.0(18.0- \\
54.0) \\
3291 / 3490 \\
(\% 94.3)\end{array}$} & & $\begin{array}{l}22.0 \\
(17.0- \\
26.0)\end{array}$ & $\begin{array}{l}88.0(60.0- \\
157.5)\end{array}$ & $\begin{array}{l}58.0(25.0- \\
109.0)\end{array}$ & \multirow[t]{3}{*}{$0.00^{\star *}$} \\
\hline & & & \multirow{2}{*}{$\begin{array}{l}1996 / 3291 \\
(\% 60.7)\end{array}$} & $\begin{array}{l}1295 / 3291 \\
(\% 39.3)\end{array}$ & & & \multirow{2}{*}{$\begin{array}{l}71 / 199(\% \\
35.7)\end{array}$} & $\begin{array}{l}128 / 199(\% \\
64.3)\end{array}$ & \multirow{2}{*}{$\begin{array}{l}199 / 3490 \\
(\% 5.7)\end{array}$} & \\
\hline & & & & 1.68 (fold) & & & & 2.32 (fold) & & \\
\hline \multirow[t]{3}{*}{$\begin{array}{l}\text { Glucose } \\
(\mathrm{mg} / \mathrm{dL})\end{array}$} & \multirow[t]{3}{*}{$60-100$} & $\begin{array}{l}56.0(31.0- \\
58.0)\end{array}$ & \multirow{3}{*}{$\begin{array}{l}90.0 \\
(84.0- \\
95.0) \\
1380 / 3504 \\
(\% 39.4)\end{array}$} & $\begin{array}{l}132.0 \\
(112.0- \\
182.0)\end{array}$ & \multirow{3}{*}{$\begin{array}{l}107.0 \\
(93.0- \\
142.0) \\
3504 / 3721 \\
(\% 94.2)\end{array}$} & $\begin{array}{l}54.0(54.0- \\
54.0)\end{array}$ & \multirow{3}{*}{$\begin{array}{l}91.0 \\
(86.0- \\
96.0) \\
35 / 217(\% \\
16.1)\end{array}$} & $\begin{array}{l}183.0 \\
(133.0- \\
260.5)\end{array}$ & $\begin{array}{l}163.0 \\
(118.0- \\
240.0)\end{array}$ & \multirow[t]{3}{*}{$0.00^{\star *}$} \\
\hline & & $0.3)$ & & $\begin{array}{l}2113 / 3504 \\
(\% \text { 60.3) }\end{array}$ & & $0.9)$ & & $\begin{array}{l}180 / 217(\% \\
82.9)\end{array}$ & $\begin{array}{l}217 / 3721 \\
(\% 5.8)\end{array}$ & \\
\hline & & 0.93 (fold) & & 1.32 (fold) & & 0.9 (fold) & & 1.83 (fold) & & \\
\hline \multirow{3}{*}{$\begin{array}{l}\mathrm{HDL} \\
(\mathrm{mg} / \mathrm{dL})\end{array}$} & \multirow[t]{3}{*}{$40-60$} & $\begin{array}{l}30.0(26.0- \\
34.0)\end{array}$ & \multirow{3}{*}{$\begin{array}{l}46.0 \\
(42.0- \\
50.0) \\
314 / 1313 \\
(\% 23.9)\end{array}$} & $\begin{array}{l}67.0(65.0- \\
71.0)\end{array}$ & $\begin{array}{l}33.0(28.0- \\
40.0)\end{array}$ & $\begin{array}{l}28.0(24.0- \\
33.0)\end{array}$ & \multicolumn{2}{|l|}{$\begin{array}{l}51.0(48.0- \\
54.5)\end{array}$} & $\begin{array}{l}33.0(26.0- \\
45.0)\end{array}$ & \multirow[t]{3}{*}{0.64} \\
\hline & & $\begin{array}{l}976 / 1313 \\
(\% 74.3)\end{array}$ & & $\begin{array}{l}23 / 1313(\% \\
1.8)\end{array}$ & $\begin{array}{l}1313 / 1366 \\
(\% 96.1)\end{array}$ & $\begin{array}{l}37 / 53(\% \\
69.8)\end{array}$ & $\begin{array}{l}16 / 53(\% \\
30.2)\end{array}$ & & $\begin{array}{l}53 / 1366(\% \\
3.9)\end{array}$ & \\
\hline & & 0.75 (fold) & & 1.12 (fold) & & 0.7 (fold) & & & & \\
\hline Calcium & $8.8-10.6$ & $\begin{array}{l}8.4(8.1- \\
8.6)\end{array}$ & $\begin{array}{l}9.30(9.0- \\
9.6)\end{array}$ & $\begin{array}{l}11.2(10.8- \\
11.4)\end{array}$ & $\begin{array}{l}8.9(8.49- \\
9.40)\end{array}$ & $\begin{array}{l}8.1(7.7- \\
8.3)\end{array}$ & $\begin{array}{l}9.2(8.91- \\
9.4)\end{array}$ & & $\begin{array}{l}8.20(7.8- \\
8.5)\end{array}$ & $0.00^{* *}$ \\
\hline & & $\begin{array}{l}1214 / 2928 \\
(\% 41.5)\end{array}$ & $\begin{array}{l}1701 / 2928 \\
(\% 58.1)\end{array}$ & $\begin{array}{l}13 / 2928(\% \\
0.4)\end{array}$ & $\begin{array}{l}2928 / 3138 \\
(\% 93.3)\end{array}$ & $\begin{array}{l}174 / 210(\% \\
82.9)\end{array}$ & $\begin{array}{l}36 / 210(\% \\
17.1)\end{array}$ & & $\begin{array}{l}210 / 3138 \\
(\% \text { 6.7) }\end{array}$ & \\
\hline & & 0.95 (fold) & & 1.06 (fold) & & 0.92 (fold) & & & & \\
\hline $\begin{array}{l}\mathrm{Cl} \\
(\mathrm{mmol} / \mathrm{L})\end{array}$ & $98-107$ & $\begin{array}{l}95.0(94.0- \\
97.0)\end{array}$ & $\begin{array}{l}103.0 \\
(101.0- \\
105.0)\end{array}$ & $\begin{array}{l}109.0 \\
(108.0- \\
110.0)\end{array}$ & $\begin{array}{l}104.0 \\
(101.0- \\
106.0)\end{array}$ & $\begin{array}{l}95.0(93.0- \\
96.0)\end{array}$ & $\begin{array}{l}103.0 \\
(101.0- \\
105.0)\end{array}$ & $\begin{array}{l}111.0 \\
(109.0- \\
1140)\end{array}$ & $\begin{array}{l}103.0(99.0- \\
108.0)\end{array}$ & 0.79 \\
\hline & & $\begin{array}{l}176 / 2497 \\
(\% 7.0)\end{array}$ & $\begin{array}{l}2021 / 2497 \\
(\% 80.9)\end{array}$ & $\begin{array}{l}300 / 2497 \\
(\% 12.0)\end{array}$ & $\begin{array}{l}2497 / 2690 \\
(\% 92.8)\end{array}$ & $\begin{array}{l}40 / 193(\% \\
20.7)\end{array}$ & $\begin{array}{l}98 / 193(\% \\
50.8)\end{array}$ & $\begin{array}{l}55 / 193(\% \\
28,5)\end{array}$ & $\begin{array}{l}193 / 2690 \\
(\% 7.2)\end{array}$ & \\
\hline & & 0.97 (fold) & & 1.02 (fold) & & $0.97(f$ & & 1.04 (fold) & & \\
\hline $\begin{array}{l}\text { T.Col. } \\
\text { (mg/dL) }\end{array}$ & $0-200$ & & $\begin{array}{l}150.0 \\
(129.0- \\
171.0)\end{array}$ & $\begin{array}{l}222.0 \\
(211.0- \\
239.0)\end{array}$ & $\begin{array}{l}158.0 \\
(134.0- \\
188.0)\end{array}$ & & $\begin{array}{l}139.0 \\
(122.0- \\
167.5)\end{array}$ & $\begin{array}{l}208.05 \\
(208.0- \\
223.0)\end{array}$ & $\begin{array}{l}153.5 \\
(126.0- \\
184.0)\end{array}$ & 0.31 \\
\hline & & & $\begin{array}{l}1106 / 1339 \\
(\% 82.6)\end{array}$ & $\begin{array}{l}233 / 1339 \\
(\% 17.4)\end{array}$ & $\begin{array}{l}1339 / 1393 \\
(\% 96.1)\end{array}$ & & $\begin{array}{l}44 / 54(\% \\
81.5)\end{array}$ & $\begin{array}{l}10 / 54(\% \\
18.5)\end{array}$ & $\begin{array}{l}54 / 1393(\% \\
3.9)\end{array}$ & \\
\hline & & & & 1.1 (fold) & & & & 1.04 (fold) & & \\
\hline $\begin{array}{l}\text { Creatinine } \\
(\mathrm{mg} / \mathrm{dL})\end{array}$ & $\begin{array}{l}\text { Male: } \\
0.84-\end{array}$ & $\begin{array}{l}0.78(0.73- \\
0.81)\end{array}$ & $\begin{array}{l}1.00 \\
(0.93- \\
1.08)\end{array}$ & $\begin{array}{l}1.47(1.35- \\
1.75)\end{array}$ & $\begin{array}{l}1.01(0.91- \\
1.15)\end{array}$ & $\begin{array}{l}0.70(0.60- \\
0.79)\end{array}$ & $\begin{array}{l}1.01 \\
(0.94- \\
1.11)\end{array}$ & $\begin{array}{l}1.82(1.42- \\
2.68)\end{array}$ & $\begin{array}{l}1.10(0.95- \\
1.46)\end{array}$ & $0.00^{* *}$ \\
\hline & 1.25 & $\begin{array}{l}212 / 1930 \\
(\% 11.0)\end{array}$ & $1394 / 1930$ & $\begin{array}{l}324 / 1930 \\
(\% 16.8)\end{array}$ & $\begin{array}{l}1930 / 2072 \\
(\% 93.1)\end{array}$ & $\begin{array}{l}15 / 142(\% \\
10.6)\end{array}$ & $75 / 142$ (\% & $\begin{array}{l}52 / 142(\% \\
36.6)\end{array}$ & $\begin{array}{l}142 / 2072 \\
(\% \text { 6.9) }\end{array}$ & \\
\hline & & 0.93 (fold) & & 1.18 (fold) & & 0.83 (fold) & & 1.46 (fold) & & \\
\hline & Female: & $\begin{array}{l}0.60(0.55- \\
0.63)\end{array}$ & 0.82 & $\begin{array}{l}1.33(1.17- \\
1.58)\end{array}$ & $\begin{array}{l}0.82(0.73- \\
0.94)\end{array}$ & $\begin{array}{l}0.49(0.48- \\
0.59)\end{array}$ & 0.86 & $\begin{array}{l}1.82(1.36- \\
2.36)\end{array}$ & $\begin{array}{l}1.02(0.83- \\
1.78)\end{array}$ & $0.00^{* *}$ \\
\hline & 1.09 & $\begin{array}{l}215 / 1946 \\
(\% 11.0)\end{array}$ & $1462 / 1946$ & $\begin{array}{l}269 / 1946 \\
(\% 13.8)\end{array}$ & $\begin{array}{l}1946 / 2037 \\
(\% 95.5)\end{array}$ & $\begin{array}{l}7 / 91(\% \\
7.7)\end{array}$ & 42/91 (\% & $\begin{array}{l}42 / 91(\% \\
46.2)\end{array}$ & $\begin{array}{l}91 / 2037(\% \\
4.5)\end{array}$ & \\
\hline & & 0.91 (fold) & & 1.22 (fold) & & 0.74 (fold) & & 1.67 (fold) & & \\
\hline
\end{tabular}

${ }^{*} \mathrm{p}<0.05,{ }^{* *} \mathrm{p}<0.01,{ }^{* *} \mathrm{p}<0.001$, $\mathrm{p}$ values denoted comparison of total median values between ICU and non-ICU groups. AIP, atherogenic index of plasma; Alb, albumin; ALP, alkaline phosphatase; ALT, alanine aminotransferase; AST, aspartate aminotransferase; Ca, calcium; Cl, chlorine; CK, creatine kinase; CKMB, creatine kinase myocardial band; D. Bil, direct bilirubin; eGFR, estimated glomerular filtration rate; GGT, gamma glutamyltransferase; HDL, high density lipoprotein; IQR, interquartile range; K, potassium; LDH, lactate dehydrogenase; LDL, low density lipoprotein; Mg, magnesium; Na, sodium; T. Bil, total bilirubin; T.Col., total cholesterol; TG, triglyceride; TIBC, total iron binding capacity; T. Protein, total protein, UA, uric acid. 


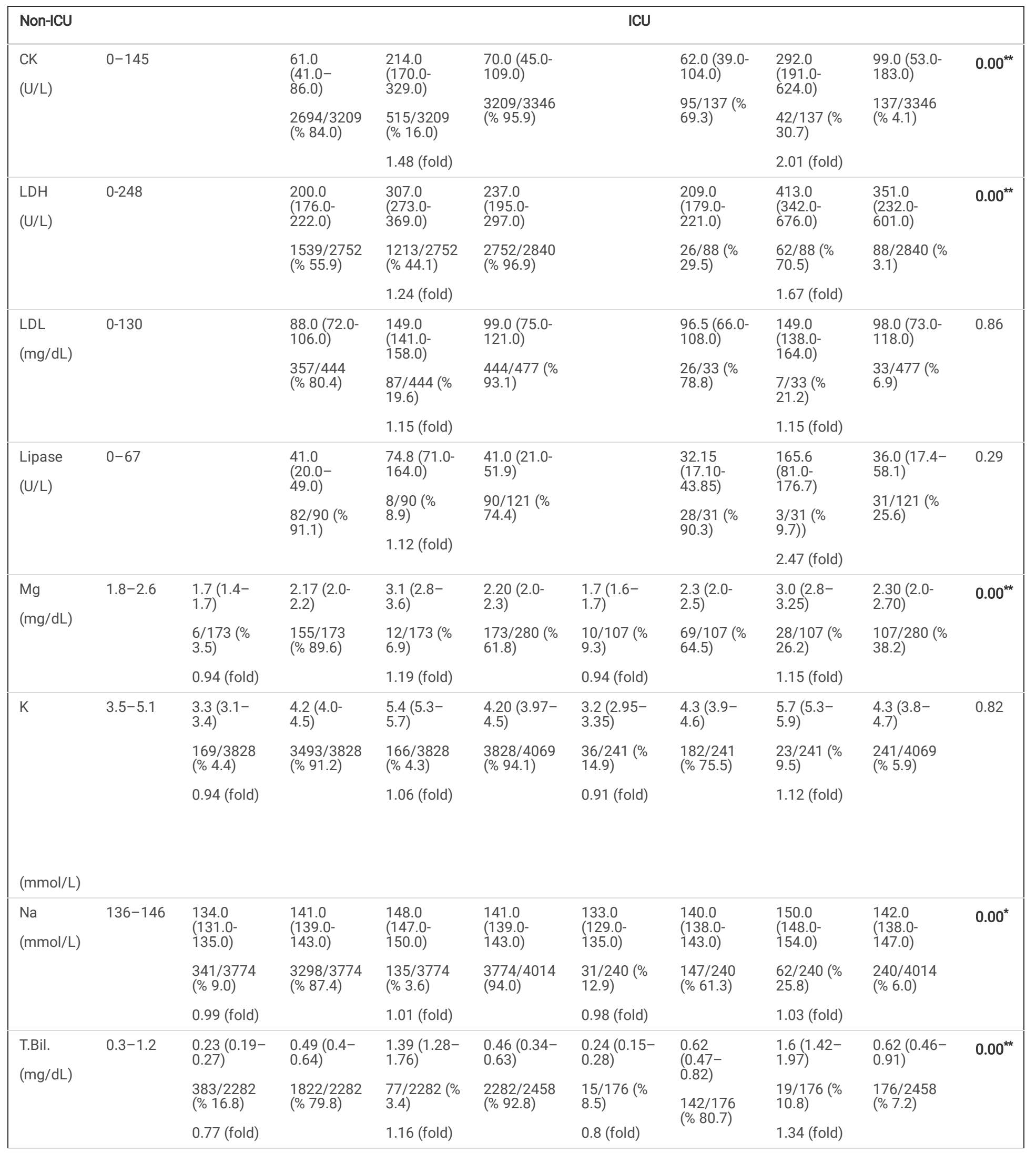

${ }^{*} \mathrm{p}<0.05,{ }^{* *} \mathrm{p}<0.01,{ }^{* * *} \mathrm{p}<0.001$, $\mathrm{p}$ values denoted comparison of total median values between ICU and non-ICU groups. AIP, atherogenic index of plasma; Alb, albumin; ALP, alkaline phosphatase; ALT, alanine aminotransferase; AST, aspartate aminotransferase; Ca, calcium; Cl, chlorine; CK, creatine kinase; CKMB, creatine kinase myocardial band; D. Bil, direct bilirubin; eGFR, estimated glomerular filtration rate; GGT, gamma glutamyltransferase; HDL, high density lipoprotein; IQR, interquartile range; K, potassium; LDH, lactate dehydrogenase; LDL, low density lipoprotein; Mg, magnesium; Na, sodium; T. Bil, total bilirubin; T.Col., total cholesterol; TG, triglyceride; TIBC, total iron binding capacity; T. Protein, total protein, UA, uric acid. 


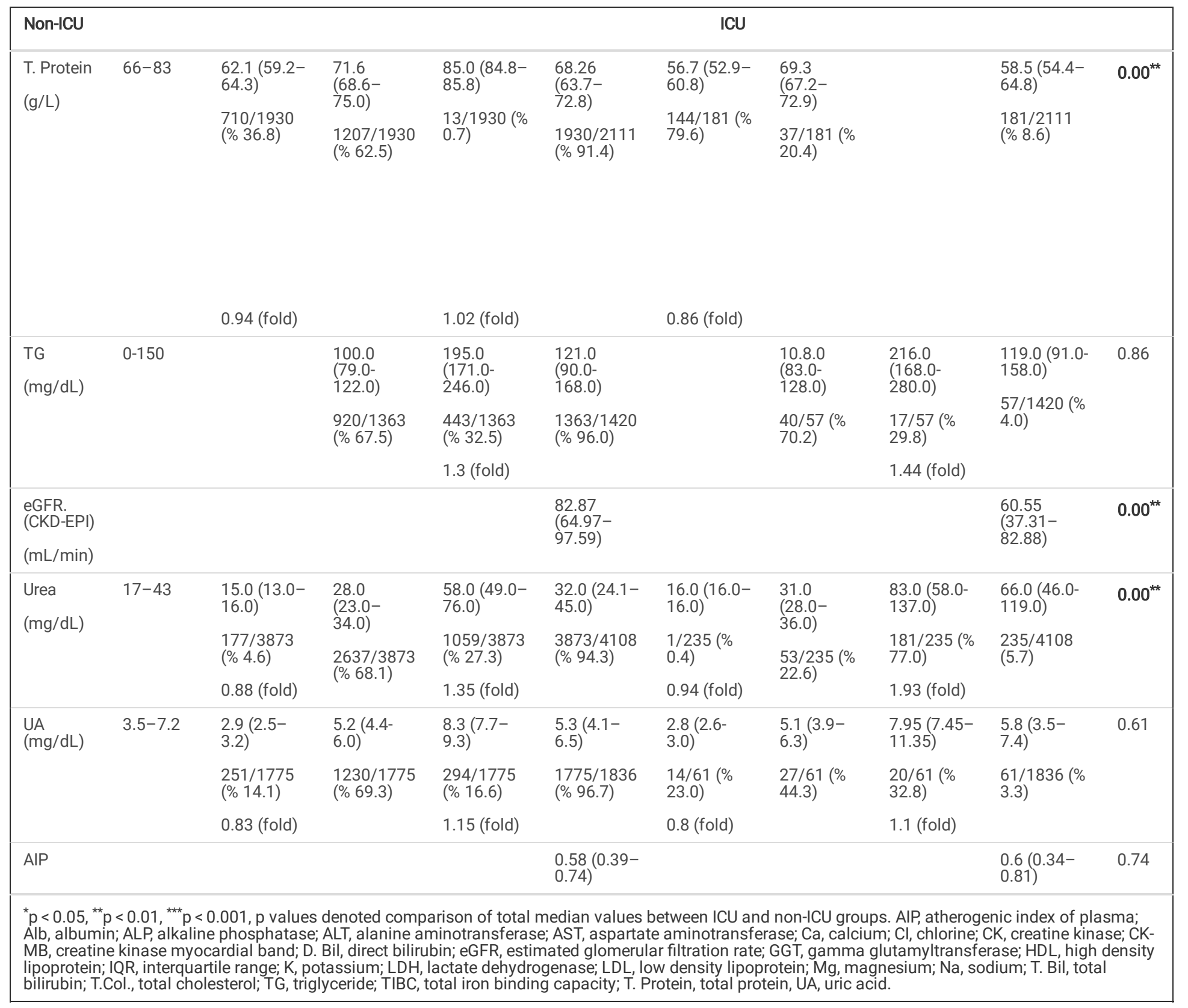

In the non-ICU group, CRP was higher in autumn than in other seasons and ferritin (only in men) gradually increased from spring to autumn while ferritin (in women) was lower in spring than other seasons. Additionally, procalcitonin was higher in summer than autumn, and ESR was higher in autumn than summer while aPTT was higher in summer than spring and was higher in spring than autumn. Moreover, LCR (lymphocyte/CRP) and SII were lower in autumn than in other seasons. In the ICU group, ferritin gradually increased from spring to autumn while procalcitonin and SII were higher in summer than autumn and ESR was higher in autumn than spring (Table 5). 
Seasonal comparison of inflammatory, cardiac and ce

\begin{tabular}{|c|c|c|c|c|c|c|c|c|c|c|c|c|c|c|}
\hline & Spring & & & & & Summer & & & & & Autumn & & & \\
\hline Test & Mean & Med & Min & Max & SD & Mean & Med & Min & Max & SD & Mean & Med & Min & Max \\
\hline CRP (mg/L) & $26.67^{\llbracket}$ & 6.44 & .15 & 284.00 & 41.99 & $25.72^{\llbracket}$ & 6.98 & 2.57 & 253.0 & 39.09 & $29.33^{*}$ & 12.40 & 3.00 & 514.1 \\
\hline $\begin{array}{l}\text { D-Dimer } \\
(\mu \mathrm{g} / \mathrm{L})\end{array}$ & 1016.1 & 494.0 & 82.0 & 19000.0 & 2128.1 & 1250.2 & 534.0 & 96.0 & 36300.0 & 3249.9 & 1068.1 & 571.9 & 1.06 & $158 \mathrm{C}$ \\
\hline Male & $229.08^{\rrbracket}$ & 140.60 & 9.60 & 1650.00 & 267.48 & $294.04^{*}$ & 193.60 & 5.80 & 2000.00 & 305.29 & $364.95^{\#}$ & 266.70 & 3.80 & 1654 \\
\hline $\begin{array}{l}\text { Ferritin } \\
(\mathrm{ml} / \mathrm{ng})\end{array}$ & $101.44^{\#}$ & 41.45 & 3.40 & 1650.00 & 192.63 & $173.30^{\star}$ & 95.35 & 2.80 & 2000.00 & 248.82 & $171.17^{\star}$ & 104.00 & 2.40 & $165 C$ \\
\hline Female & & & & & & & & & & & & & & \\
\hline $\begin{array}{l}\text { Fibrinogen } \\
\text { (mg/dL) }\end{array}$ & 327.9 & 324.4 & 153.7 & 501.3 & 63.3 & 339.3 & 327.8 & 125.5 & 509.5 & 76.6 & 337.8 & 331.3 & 70.6 & 681. \\
\hline INR & 1.94 & 1.11 & .77 & 108.0 & 9.24 & 1.16 & 1.1 & .8 & 6.2 & .32 & 1.18 & 1.10 & .87 & 7.57 \\
\hline PT (second) & 14.45 & 13.2 & 9.40 & 129.0 & 10.4 & 13.7 & 13.0 & 10.0 & 67.3 & 3.44 & 13.98 & 13.1 & 10.5 & 81.01 \\
\hline $\begin{array}{l}\text { Procalcitonin } \\
\text { (ng/mL) }\end{array}$ & $0.88^{\#^{*}}$ & 0.12 & 0.05 & 27.00 & 3.3 & $4.51^{\#}$ & 0.12 & 0.12 & 87.0 & 12.1 & $.46^{*}$ & .12 & .12 & 100.1 \\
\hline $\begin{array}{l}\text { ESR } \\
\text { (mm/hour) }\end{array}$ & $27.36^{\square^{\star}}$ & 22.0 & 2.00 & 120.0 & 23.8 & $22.9^{\bigotimes}$ & 18.0 & 2.00 & 120.0 & 20.07 & $30.8^{\star}$ & 22.0 & 2.00 & 139.1 \\
\hline $\begin{array}{l}\text { Troponin I } \\
\text { (ng/L) }\end{array}$ & 14.26 & 10.0 & 10.00 & 680.0 & 40.0 & 18.5 & 10.0 & 10.00 & 3300.0 & 129.97 & 26.18 & 10.00 & 1.00 & $460 C$ \\
\hline $\begin{array}{l}\text { aPTT } \\
\text { (second) }\end{array}$ & $35.65^{\#}$ & 31.4 & 20.2 & 414.0 & 30.2 & $37.9^{\bigotimes}$ & 35.25 & 12.0 & 101.0 & 10.89 & $35.0^{*}$ & 33.0 & 12.0 & 353.1 \\
\hline LCR & $0.32^{*}$ & 0.18 & 0.00 & 12.42 & 0.79 & $0.28^{*}$ & 0.17 & 0.00 & 6.0 & 0.42 & $0.22^{\rrbracket}$ & 0.11 & 0.00 & 3.15 \\
\hline SII & $1364.6^{囚}$ & 671.7 & 9.27 & 14203.0 & 1708.8 & $1386.1^{\bigotimes}$ & 622.3 & 10.2 & 18480.8 & 1945.1 & $929.6^{*}$ & 501.7 & 6.96 & $229 \epsilon$ \\
\hline
\end{tabular}

${ }^{*} \#$ \#. Mean values with the same sign are similar, those with different signs have a statistically significant difference. ${ }^{*} p<0.05{ }^{* *} p<0.01,{ }^{* * *} p<0.001, p$ value: lymphocyte to C-reactive protein ratio; Max, maximum; Med, median; Min, minimum; SD, standard deviation; PT, prothrombin time; SII, systemic immune-infla 
Table 6

Comparison oof inflammatory, cardiac and coagulation biomarkers in the intensive care unit (ICU) and non-ICU groups on admission.

\begin{tabular}{|c|c|c|c|c|c|c|c|c|c|c|}
\hline & & Non-ICU & & & & ICU & & & & \\
\hline \multirow[t]{2}{*}{ Test } & \multirow{2}{*}{$\begin{array}{l}\text { Reference } \\
\text { Range }\end{array}$} & Decreased & Normal & Increased & Total & Decreased & Normal & Increased & Total & $p$ \\
\hline & & $\begin{array}{l}\text { Median } \\
(\text { IQR) } n / n \\
(\%)\end{array}$ & $\begin{array}{l}\text { Median } \\
\text { (IQR) } n / n \\
(\%)\end{array}$ & $\begin{array}{l}\text { Median } \\
\text { (IQR) } n / n \\
(\%)\end{array}$ & $\begin{array}{l}\text { Median } \\
\text { (IQR) } n / n \\
(\%)\end{array}$ & $\begin{array}{l}\text { Median } \\
\text { (IQR) } n / n \\
(\%)\end{array}$ & $\begin{array}{l}\text { Median } \\
\text { (IQR) } n / n \\
(\%)\end{array}$ & $\begin{array}{l}\text { Median } \\
\text { (IQR) } n / n \\
(\%)\end{array}$ & $\begin{array}{l}\text { Median } \\
\text { (IQR) } n / n \\
(\%)\end{array}$ & \\
\hline \multirow[t]{3}{*}{ CRP (mg/L) } & \multirow[t]{3}{*}{$0-5$} & \multirow[t]{3}{*}{-} & $\begin{array}{l}3.02(3.02- \\
3.14)\end{array}$ & $\begin{array}{l}24.30 \\
(11.40- \\
55.75)\end{array}$ & $\begin{array}{l}10.20 \\
(3.14-34.0)\end{array}$ & \multirow[t]{3}{*}{-} & $\begin{array}{l}3.02 \\
(3.02- \\
3.02)\end{array}$ & $\begin{array}{l}81.50 \\
(37.00- \\
139.00)\end{array}$ & $\begin{array}{l}79.0 \\
(30.05- \\
138.0)\end{array}$ & \multirow[t]{3}{*}{$0.00^{*}$} \\
\hline & & & \multirow[t]{2}{*}{$\begin{array}{l}1378 / 3610 \\
(\% 38.2)\end{array}$} & $\begin{array}{l}2232 / 3610 \\
(\% \text { 61.8) }\end{array}$ & $\begin{array}{l}3610 / 3800 \\
(\% 95.0)\end{array}$ & & \multirow[t]{2}{*}{$\begin{array}{l}17 / 190(\% \\
8.9)\end{array}$} & $\begin{array}{l}173 / 190(\% \\
91.1)\end{array}$ & \multirow[t]{2}{*}{$\begin{array}{l}190 / 3800 \\
(\% 5.0)\end{array}$} & \\
\hline & & & & 4.86 (fold) & & & & 16.3 (fold) & & \\
\hline \multirow[t]{3}{*}{$\begin{array}{l}\text { D-Dimer } \\
(\mu \mathrm{g} / \mathrm{L})\end{array}$} & \multirow[t]{3}{*}{$80-583$} & $\begin{array}{l}45.77 \\
(32.99- \\
64.93)\end{array}$ & $\begin{array}{l}341.06 \\
(241.0- \\
444.0)\end{array}$ & $\begin{array}{l}993.5 \\
(739.2- \\
1665.0)\end{array}$ & $\begin{array}{l}541.8 \\
(320.0- \\
954.0)\end{array}$ & \multirow[t]{3}{*}{-} & $\begin{array}{l}371.0 \\
(353.0- \\
408.0)\end{array}$ & $\begin{array}{l}1640.0 \\
(1082.0- \\
4495.2)\end{array}$ & $\begin{array}{l}1365.0 \\
(989.1- \\
3982.0)\end{array}$ & \multirow[t]{3}{*}{$0.00^{*}$} \\
\hline & & $\begin{array}{l}20 / 1651(\% \\
1.2)\end{array}$ & $\begin{array}{l}862 / 1651 \\
(\% 52.2)\end{array}$ & $\begin{array}{l}769 / 1651 \\
(\% 46.6)\end{array}$ & $\begin{array}{l}1651 / 1710 \\
(\% 96.5)\end{array}$ & & $\begin{array}{l}5 / 59(\% \\
8.5)\end{array}$ & $\begin{array}{l}54 / 59(\% \\
91.5)\end{array}$ & $\begin{array}{l}59 / 1710(\% \\
3.5)\end{array}$ & \\
\hline & & 0.57 (fold) & & 1.70 (fold) & & & & 2.81 (fold) & & \\
\hline \multirow{8}{*}{$\begin{array}{l}\text { Ferritin } \\
(\mathrm{ml} / \mathrm{ng})\end{array}$} & \multirow{4}{*}{$\begin{array}{l}\text { Male } \\
22-322\end{array}$} & \multirow{2}{*}{$\begin{array}{l}14.70 \\
(10.70- \\
19.30)\end{array}$} & \multirow{2}{*}{$\begin{array}{l}130.10 \\
(79.2- \\
213.4)\end{array}$} & \multirow{2}{*}{$\begin{array}{l}541.7 \\
(410.7- \\
776.0)\end{array}$} & \multirow{2}{*}{$\begin{array}{l}220.9 \\
(101.5- \\
434.3)\end{array}$} & \multirow{2}{*}{$\begin{array}{l}10.10 \\
(4.22- \\
16.30)\end{array}$} & \multirow{2}{*}{$\begin{array}{l}188.9 \\
(135.7- \\
242.2)\end{array}$} & \multirow{2}{*}{$\begin{array}{l}855.3 \\
(540.3- \\
1160.6)\end{array}$} & \multirow{2}{*}{$\begin{array}{l}570.6 \\
(294.6- \\
1050.9)\end{array}$} & \multirow[t]{4}{*}{$0.00^{*}$} \\
\hline & & & & & & & & & & \\
\hline & & $\begin{array}{l}97 / 2705(\% \\
3.6)\end{array}$ & $\begin{array}{l}1631 / 2705 \\
(\% 60.3)\end{array}$ & $\begin{array}{l}977 / 2705 \\
(\% 36.1)\end{array}$ & $\begin{array}{l}2705 / 2806 \\
(\% 96.4)\end{array}$ & $\begin{array}{l}3 / 101(\% \\
3.0)\end{array}$ & $\begin{array}{l}25 / 101( \\
\% 24.8)\end{array}$ & $\begin{array}{l}73 / 101(\% \\
72.3)\end{array}$ & $\begin{array}{l}101 / 2806 \\
(\% 3.6)\end{array}$ & \\
\hline & & 0.67 (fold) & & 1.68 (fold) & & 0.46 (fold) & & 2.67 (fold) & & \\
\hline & \multirow{4}{*}{$\begin{array}{l}\text { Female } \\
10-291\end{array}$} & $\begin{array}{l}6.75(5.10- \\
8.30)\end{array}$ & $\begin{array}{l}81.10 \\
(33.60-\end{array}$ & $\begin{array}{l}454.05 \\
(349.50-\end{array}$ & $\begin{array}{l}90.50 \\
(32.0-\end{array}$ & - & $\begin{array}{l}117.90 \\
(38.4-\end{array}$ & 529.85 & 207.9 & $0.00^{*}$ \\
\hline & & $82 / 1311$ \% & 150.50) & 654.0) & 197.2) & & 171.0) & $706.9)$ & 463.8) & \\
\hline & & $6.3)$ & $\begin{array}{l}1039 / 1311 \\
(\% 79.3)\end{array}$ & $\begin{array}{l}190 / 1311 \\
(\% 14.5)\end{array}$ & $\begin{array}{l}1311 / 1372 \\
(\% 95.6)\end{array}$ & & $\begin{array}{l}35 / 61(\% \\
57.4)\end{array}$ & $\begin{array}{l}26 / 61(\% \\
42.6)\end{array}$ & $\begin{array}{l}61 / 1372(\% \\
4.4)\end{array}$ & \\
\hline & & 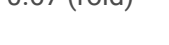 & & 1.56 (fold) & & & & 1.82 (fold) & & \\
\hline $\begin{array}{l}\text { Fibrinogen } \\
(\mathrm{mg} / \mathrm{dL})\end{array}$ & $180-450$ & $\begin{array}{l}160.66 \\
(134.71- \\
172.56)\end{array}$ & $\begin{array}{l}321.10 \\
(290.70- \\
357.52)\end{array}$ & $\begin{array}{l}486.28 \\
(472.56- \\
501.36)\end{array}$ & $\begin{array}{l}327.8 \\
(293.7- \\
370.35)\end{array}$ & $\begin{array}{l}137.6 \\
(137.6- \\
137.6)\end{array}$ & $\begin{array}{l}353.5 \\
(317.9- \\
379.1)\end{array}$ & $\begin{array}{l}480.0 \\
(466.15- \\
493.64)\end{array}$ & $\begin{array}{l}359.7 \\
(321.1- \\
392.5)\end{array}$ & $0.00^{*}$ \\
\hline & & $\begin{array}{l}24 / 2260(\% \\
1.1)\end{array}$ & $\begin{array}{l}2039 / 2260 \\
(\% 90.2)\end{array}$ & $\begin{array}{l}197 / 2260 \\
(\% 8.7)\end{array}$ & $\begin{array}{l}2263 / 2369 \\
(\% 95.4)\end{array}$ & $\begin{array}{l}1 / 109(\% \\
0.9)\end{array}$ & $\begin{array}{l}98 / 109(\% \\
89.9)\end{array}$ & $\begin{array}{l}10 / 109(\% \\
9.2)\end{array}$ & $\begin{array}{l}109 / 2369 \\
(\% 4.6)\end{array}$ & \\
\hline & & 0.89 (fold) & & 1.08 (fold) & & 0.76 (fold) & & 1.07 (fold) & & \\
\hline INR & $0.6-1.2$ & - & $\begin{array}{l}1.08(1.04- \\
1.13)\end{array}$ & $\begin{array}{l}1.31(1.25- \\
1.46)\end{array}$ & $\begin{array}{l}1.10 \\
(1.04-\end{array}$ & - & $\begin{array}{l}1.12 \\
(1.08-\end{array}$ & $\begin{array}{l}1.38(1.26- \\
1.66)\end{array}$ & $\begin{array}{l}1.20(1.12- \\
1.38)\end{array}$ & $0.00^{*}$ \\
\hline & & & $\begin{array}{l}1161 / 1426 \\
(\% 81.4)\end{array}$ & $\begin{array}{l}265 / 1426 \\
(\% 18.6)\end{array}$ & $1426 / 1561$ & & 68/135 (\% & $\begin{array}{l}67 / 135(\% \\
49.6)\end{array}$ & $\begin{array}{l}135 / 1561 \\
(\% 8.6)\end{array}$ & \\
\hline & & & & 1.09 (fold) & $(\%$ & & 50. & 1.15 (fold) & & \\
\hline PT (second) & $\begin{array}{l}10.1- \\
15.9\end{array}$ & $\begin{array}{l}9.70(9.40- \\
10.0)\end{array}$ & $\begin{array}{l}13.00 \\
(12.4-13.7)\end{array}$ & $\begin{array}{l}17.8(16.6- \\
21.6)\end{array}$ & $\begin{array}{l}13.1 \\
(12.5- \\
139)\end{array}$ & - & $\begin{array}{l}13.90 \\
(13.00- \\
14.70)\end{array}$ & $\begin{array}{l}18.9 \\
(17.10- \\
22.40)\end{array}$ & $\begin{array}{l}14.3(13.2- \\
16.0)\end{array}$ & $0.00^{*}$ \\
\hline & & $\begin{array}{l}2 / 1426 \\
(0.1)\end{array}$ & $\begin{array}{l}1311 / 1426 \\
(\% 91.9)\end{array}$ & $\begin{array}{l}113 / 1426 \\
(\% 7.9)\end{array}$ & $1426 / 1561$ & & $100 / 135$ & $35 / 135$ (\% & $\begin{array}{l}135 / 1561 \\
(\% 8.6)\end{array}$ & \\
\hline & & 0.96 (fold) & & 1.12 (fold) & & & & & & \\
\hline & & & & & & & & 1.19 (fold) & & \\
\hline
\end{tabular}

${ }^{*} \mathrm{p}<0.001$, p values denoted comparison of total median values between ICU and non-ICU groups. aPTT, activated partial prothrombin time; CRP, C-reactive protein; ESR, erythrocyte sedimentation rate; INR, international normalized ratio; IQR, interquartile range; LCR, lymphocyte to C-reactive protein ratio; Max, maximum; Med, median; Min, minimum; SD, standard deviation; PT, prothrombin time; SII, systemic immune-inflammation index. 


\begin{tabular}{|c|c|c|c|c|c|c|c|c|c|c|}
\hline & & Non-ICU & & & & ICU & & & & \\
\hline \multirow[t]{3}{*}{$\begin{array}{l}\text { Procalcitonin } \\
(\mathrm{ng} / \mathrm{mL})\end{array}$} & \multirow[t]{3}{*}{$<0.15$} & \multirow[t]{3}{*}{-} & $\begin{array}{l}0.12(0.12- \\
0.12)\end{array}$ & $\begin{array}{l}0.35(0.20- \\
12.00)\end{array}$ & \multirow[t]{3}{*}{$\begin{array}{l}0.12 \\
(0.12- \\
0.12)\end{array}$} & \multirow[t]{3}{*}{ - } & \multirow{3}{*}{$\begin{array}{l}0.12 \\
(0.12- \\
0.12) \\
29 / 105(\% \\
27.6)\end{array}$} & $\begin{array}{l}1.5(0.32- \\
14.00)\end{array}$ & \multirow[t]{3}{*}{$\begin{array}{l}0.39(0.13- \\
6.5)\end{array}$} & \multirow[t]{3}{*}{$0.00^{*}$} \\
\hline & & & \multirow[t]{2}{*}{$\begin{array}{l}1832 / 2337 \\
(\% 78.4)\end{array}$} & $\begin{array}{l}505 / 2337 \\
(\% 21.6)\end{array}$ & & & & & & \\
\hline & & & & 2.33 (fold) & & & & 10 (fold) & & \\
\hline \multirow[t]{3}{*}{$\begin{array}{l}\text { ESR } \\
\text { (mm/hour) }\end{array}$} & \multirow[t]{3}{*}{$0-20$} & \multirow[t]{3}{*}{-} & \multirow{3}{*}{$\begin{array}{l}10.00 \\
(4.00- \\
15.00) \\
\\
941 / 1846 \\
(\% 51.0)\end{array}$} & $\begin{array}{l}41.0(31.0- \\
60.0)\end{array}$ & \multirow{3}{*}{$\begin{array}{l}20.0 \\
(10.0- \\
41.0) \\
1846 / 1944 \\
(\% 95.0)\end{array}$} & \multirow[t]{3}{*}{-} & \multirow{3}{*}{$\begin{array}{l}9.50 \\
(4.00- \\
14.00) \\
26 / 98(\% \\
26.5)\end{array}$} & $\begin{array}{l}58.00 \\
(39.00- \\
78.50)\end{array}$ & \multirow{3}{*}{$\begin{array}{l}45.5(18.0- \\
68.0) \\
98 / 1944(\% \\
5.0)\end{array}$} & \multirow[t]{3}{*}{$0.00^{*}$} \\
\hline & & & & $\begin{array}{l}905 / 1846 \\
(\% 49.0)\end{array}$ & & & & $\begin{array}{l}72 / 98(\% \\
73.5)\end{array}$ & & \\
\hline & & & & 2.05 (fold) & & & & 2.9 (fold) & & \\
\hline \multirow{5}{*}{$\begin{array}{l}\text { Troponin I } \\
\text { (ng/L) }\end{array}$} & \multirow[t]{5}{*}{$10-23$} & $\begin{array}{l}1.0(1.0- \\
1.0)\end{array}$ & $\begin{array}{l}10.0(10.0- \\
10.0)\end{array}$ & $\begin{array}{l}54.5(41.5- \\
130.0)\end{array}$ & \multirow{3}{*}{$\begin{array}{l}10.0 \\
(10.0- \\
10.0)\end{array}$} & \multirow[t]{5}{*}{-} & \multirow{3}{*}{$\begin{array}{l}10.0 \\
(10.0- \\
10.0)\end{array}$} & \multirow{3}{*}{$\begin{array}{l}135.0 \\
(54.0- \\
360.0)\end{array}$} & $\begin{array}{l}10.0(10.0- \\
51.0)\end{array}$ & \multirow[t]{5}{*}{$0.00^{\star}$} \\
\hline & & $\begin{array}{l}1 / 2102 \\
(0.0)\end{array}$ & $\begin{array}{l}2020 / 2102 \\
(\% 96.1)\end{array}$ & $\begin{array}{l}81 / 2102(\% \\
3.9)\end{array}$ & & & & & $\begin{array}{l}110 / 2212 \\
(\% 5.0)\end{array}$ & \\
\hline & & 0.1 (fold) & & 2.37 (fold) & & & & & & \\
\hline & & & & & \multirow[t]{2}{*}{$\begin{array}{l}2102 / 2212 \\
(\% 95.0)\end{array}$} & & \multirow[t]{2}{*}{$\begin{array}{l}73 / 110(\% \\
66.4)\end{array}$} & $\begin{array}{l}37 / 110(\% \\
33.6)\end{array}$ & & \\
\hline & & & & & & & & 5.87 (fold) & & \\
\hline \multirow[t]{3}{*}{$\begin{array}{l}\text { aPTT } \\
\text { (second) }\end{array}$} & \multirow[t]{3}{*}{$22-45$} & $\begin{array}{l}20.10 \\
(14.90- \\
20.90)\end{array}$ & \multirow{3}{*}{$\begin{array}{l}32.8(29.5- \\
36.5) \\
1201 / 1357 \\
(\% 88.5)\end{array}$} & $\begin{array}{l}52.75 \\
(48.5-59.0)\end{array}$ & \multirow{3}{*}{$\begin{array}{l}33.4 \\
(29.9- \\
38.4) \\
1357 / 1475 \\
(\% 92.0)\end{array}$} & \multirow{3}{*}{$\begin{array}{l}12.0 \\
(12.0- \\
12.0) \\
2 / 118 \text { (\% } \\
1.7) \\
0.54 \text { (fold) }\end{array}$} & \multirow{3}{*}{$\begin{array}{l}33.3 \\
(29.2- \\
37.3) \\
103 / 118 \\
(\% 87.3)\end{array}$} & $\begin{array}{l}51.7(47.8- \\
56.6)\end{array}$ & $\begin{array}{l}34.5(29.4- \\
40.2)\end{array}$ & 0.88 \\
\hline & & $\begin{array}{l}8 / 1357(\% \\
0.6)\end{array}$ & & $\begin{array}{l}148 / 1357 \\
(\% \text { 10.9) }\end{array}$ & & & & $\begin{array}{l}13 / 118(\% \\
11.0)\end{array}$ & $\begin{array}{l}118 / 1475 \\
(\% 8.0)\end{array}$ & \\
\hline & & 0.91 (fold) & & $1.1 /$ (fold) & & & & 1.15 (fold) & & \\
\hline LCR & - & - & - & - & $\begin{array}{l}0.13 \\
(0.04- \\
0.36)\end{array}$ & - & - & - & $\begin{array}{l}0.02(0.01- \\
0.04)\end{array}$ & $0.00^{*}$ \\
\hline SII & & & & & $\begin{array}{l}547.0 \\
(316.0- \\
1161.3)\end{array}$ & & & & $\begin{array}{l}609.0 \\
(336.1- \\
1428 . .7)\end{array}$ & 0.10 \\
\hline
\end{tabular}

CRP, D-dimer, ferritin, fibrinogen, international normalized ratio (INR), PT, procalcitonin, ESR and troponin were higher and LCR was lower in the ICU group compared to the non-ICU group (Table S1).

\section{Discussion}

While COVID-19 continues to rapidly spread in the world, the disease and mortality ratios are increasing. Unfortunately, the contamination has not been prevented as an effective treatment for the disease is yet to be found [1]. The vaccination has begun but it is too soon to see its effect. On the other hand, important mutations have been detected in the structure of the virus while the disease continues to spread [6, 7]. Therefore, it is possible that COVID-19 will be in our lives for a long time. It is essential to explain this disease with all its aspects and to reveal the differences that may develop in time. In this regard, this study is important as it shows the timely changes in routine blood biomarkers in COVID-19. Also, these changes are important for the diagnosis of the disease but the fact that the tests' results changed negatively indicates that the severity of the disease increased in time.

This study showed that advanced age and male individuals stay are hospitalized in the intensive care unit more. It is known that advanced age is a bad prognostic indicator [14]. The increased MPV, NLR, dNLR and decreased eosinophil, hematocrit, hemoglobin, lymphocyte, RBC values in autumn, the third period of the disease, showed that the disease progresses more severely than the early period. It was reported that NLR is an independent risk factor for mortality [15]. These results might be effective in conditions such as the increased possibility of infection of the elderly and people with chronic diseases than the early days of the disease. Increased glucose, creatinine, urea, uric acid, ALT, AST, GGT and decreased albumin, ALP, calcium, total bilirubin, total protein and eGFR in autumn show that organ damage such as the pancreas, kidney and live damage is more in these patients. Additionally, the mutations in the virus structure or changes in the clinical characteristics of the disease might have caused this result. The result that CRP, ferritin, ESR were higher in autumn while 
LCR was lower shows that the patients produced a more powerful inflammatory response in this last period. Previous studies reported that increased CRP, ESR, LDH and decreased albumin, eosinophil, and lymphocyte are the most important laboratory findings of the disease $[9,10,13]$.

While the results that MCV, neutrophil, plateletcrit, PDW, RDW, WBC, P-LRC, NLR, d-NLR and PLR were higher and eosinophil, hematocrit, hemoglobin, lymphocyte, $\mathrm{MCHC}$, monocyte, $\mathrm{RBC}$ and LMR were lower in the ICU group were expected, they indicate that the immune response is defective in this group, that lymphocytic and erythrocyte series in the bone marrow might have been suppressed and there might be platelet and erythrocyte deformities. Lymphopenia is the most important laboratory findings that show defective immune response and risk of hospitalization in the intensive care unit in COVID-19 [9]. Neutrophil might be related to hyperinflammation and cytokine storm or superimposed bacterial infection [16]. NLR shows the severity of inflammation and it is a powerful predictive and prognostic indicator [11, 17]. Also, the PLR value is asserted to be correlated with the severity of cytokine storm [18]. It was asserted that eosinopenia can be used in diagnosis in combination with lymphopenia [19]. Additionally, changes in the RBC parameters are related to distorted erythropoiesis [8]. High RDW is reported to be related to high mortality [20].

Moreover, the result that ALT, AST, AST/ALT, ALP, CK-MB, direct bilirubin, GGT, glucose, creatinine, CK, LDH, magnesium, sodium, total bilirubin, urea and troponin values were high while albumin, iron, calcium, total protein and eGFR were low in the ICU group indicates that this group had more muscle, liver, cardiac, pancreas, lung and kidney pathologies. This multi-organ involvement in COVID-19 was associated with the widespread distribution of angiotensinconverting enzyme (ACE2) receptors in the body [21]. High creatinine, AST, LDH, troponin, CRP, ferritin, procalcitonin, D-dimer, and fibrinogen values were reported to be the indicator of clinical deterioration and mortality in these patients [22, 23]. It is asserted that live function tests such as ALT, AST, GGT, ALP and bilirubin often increase in COVID-19 but they do not require treatment and the underlying reasons for these increased values are hyper inflammation and thrombotic microangiopathy [24]. The ratio of AST/ALT (de ritis ratio) was found to be an important indicator especially in women [25]. It was reported that fasting hyperglycemia increases mortality as well as the development of complications [26]. It was stated that sodium, potassium and calcium are low in severe COVID-19 patients; thus, electrolytes should be monitored [27]. The reason for high sodium in the ICU group in the present study might have been iatrogenic.

Additionally, increased CRP, ferritin, procalcitonin, ESR and decreased LCR values in the ICU group show that this group had a more powerful inflammatory response. CRP is a sensitive indicator of inflammation and tissue damage and it increases in most COVID-19 patients [28, 29]. Previous studies reported that high CRP and LDH and lymphopenia may show the necessity to transfer the patient to intensive care [10]. It is asserted that low LCR is an indicator of high mortality and the need for mechanical ventilation [30]. A high level of procalcitonin might be due to secondary bacterial infection [12]. Increased d-Dimer and fibrinogen are the indicators of intravascular blood clot coagulation and high PT and INR values. Therefore, these patients should be monitored in terms of the development of disseminated intravascular coagulopathy (DIC) and acute respiratory distress (ARDS) [12, 21, 31].

Another new information provided by this study is that the ratio of the changes in routine blood biomarkers was revealed clearly. Accordingly, the increases in these tests were mostly lower than twice while decreases were lower than half (bigger than 0.5 times). Thus, even small changes in the laboratory tests of these patients must be considered.

The limitations of this study are that it was designed retrospectively and single-center and that comorbidities and medications received were not included in the study.

In conclusion, this study showed that the changes in routine laboratory tests in COVID-19 vary in the period from the emergence of the disease until now. There were increases in some tests, decreases in some and similarities in some during this period. Thus, the timely changes in the routine laboratory tests in COVID19 should be checked with new studies. Also, the timely change of routine laboratory tests was mostly more negative in this study and this indicates that the disease progresses more severely compared to the period when it first emerged.

\section{Declarations}

\section{Competing interests:}

The authors declare no competing interests.

\section{References}

1. WHO. No Title, https://covid19.who.int/? gclid=CjwKCAiAgJWABhArEiwAmNVTB5Zoc03kqun6wvZFTCPYKHDJgnmRnKi7H986LHwioRh_hITAqppXgBoCQPMQAvD_BwE (accessed 19 January 2021).

2. Yang X-D, Li H-L, Cao Y-E. Influence of Meteorological Factors on the COVID-19 Transmission with Season and Geographic Location. Int J Environ Res Public Health; 18. Epub ahead of print 2021. DOI: 10.3390/ijerph18020484.

3. Adedokun KA, Olarinmoye AO, Olarinmoye AO, et al. A close look at the biology of SARS-CoV-2, and the potential influence of weather conditions and seasons on COVID-19 case spread. Infect Dis Poverty 2020; 9: 1-5.

4. Méndez-Arriaga F. The temperature and regional climate effects on communitarian COVID-19 contagion in Mexico throughout phase 1. Sci Total Environ. Epub ahead of print 2020. DOI: 10.1016/j.scitotenv.2020.139560.

5. Mattiuzzi C, Henry BM, Sanchis-Gomar F, et al. Association between climate and new daily diagnoses of COVID-19. medRxiv 2020; 2020.11.12.20230888.

6. Korber B, Fischer WM, Gnanakaran S, et al. Tracking Changes in SARS-CoV-2 Spike: Evidence that D614G Increases Infectivity of the COVID-19 Virus. Cell 2020; 182: 812-827.e19.

Page 18/19 
7. van Dorp L, Acman M, Richard D, et al. Emergence of genomic diversity and recurrent mutations in SARS-CoV-2. Infect Genet Evol. Epub ahead of print 2020. DOI: 10.1016/j.meegid.2020.104351.

8. Khartabil TA, Russcher $\mathrm{H}$, van der Ven A, et al. A summary of the diagnostic and prognostic value of hemocytometry markers in COVID-19 patients. Crit Rev Clin Lab Sci 2020; 57: 415-431.

9. Mertoglu C, Huyut MT, Arslan Y, et al. How do routine laboratory tests change in coronavirus disease 2019? Scand J Clin Lab Invest. Epub ahead of print 2020. DOI: $10.1080 / 00365513.2020 .1855470$.

10. Zhang ZL, Hou YL, Li DT, et al. Laboratory findings of COVID-19: a systematic review and meta-analysis. Scandinavian Journal of Clinical and Laboratory Investigation. Epub ahead of print 2020. DOI: 10.1080/00365513.2020.1768587.

11. Khartabil TA, Russcher H, van der Ven A, et al. A summary of the diagnostic and prognostic value of hemocytometry markers in COVID-19 patients. Critical Reviews in Clinical Laboratory Sciences. Epub ahead of print 2020. DOI: 10.1080/10408363.2020.1774736.

12. Terpos E, Ntanasis-Stathopoulos I, Elalamy I, et al. Hematological findings and complications of COVID-19. Am J Hematol 2020; 95: 834-847.

13. Pourbagheri-Sigaroodi A, Bashash D, Fateh F, et al. Laboratory findings in COVID-19 diagnosis and prognosis. Clin Chim Acta 2020; 510: 475-482.

14. Verity R, Okell LC, Dorigatti I, et al. Estimates of the severity of coronavirus disease 2019: a model-based analysis. Lancet Infect Dis. Epub ahead of print 2020. DOI: $10.1016 /$ S1473-3099(20)30243-7.

15. Liu Y, Du X, Chen J, et al. Neutrophil-to-lymphocyte ratio as an independent risk factor for mortality in hospitalized patients with COVID-19. J Infect. Epub ahead of print 2020. DOI: 10.1016/j.jinf.2020.04.002.

16. Frater JL, Zini G, d'Onofrio G, et al. COVID-19 and the clinical hematology laboratory. Int J Lab Hematol 2020; 42: 11-18.

17. Kerboua KE. NLR: A Cost-effective Nomogram to Guide Therapeutic Interventions in COVID-19. Immunol Invest 2020; 00: 1-9.

18. Qu R, Ling Y, Zhang Y hui zhi, et al. Platelet-to-lymphocyte ratio is associated with prognosis in patients with coronavirus disease-19. $J$ Med Virol. Epub ahead of print 2020. DOI: 10.1002/jmv.25767.

19. Zhang J jin, Dong X, Cao Y yuan, et al. Clinical characteristics of 140 patients infected with SARS-CoV-2 in Wuhan, China. Allergy Eur J Allergy Clin Immunol 2020; 1-12.

20. Foy BH, Carlson JCT, Reinertsen E, et al. Association of Red Blood Cell Distribution Width With Mortality Risk in Hospitalized Adults With SARS-CoV-2 Infection. JAMA Netw open. Epub ahead of print 2020. DOI: 10.1001/jamanetworkopen.2020.22058.

21. Fang B, Meng QH. The laboratory's role in combating COVID-19. Crit Rev Clin Lab Sci 2020; 57: 400-414.

22. Cecconi M, Piovani D, Brunetta E, et al. Early Predictors of Clinical Deterioration in a Cohort of 239 Patients Hospitalized for Covid-19 Infection in Lombardy, Italy. J Clin Med 2020; 9: 1548.

23. Aboughdir M, Kirwin T, Khader AA, et al. Prognostic value of cardiovascular biomarkers in COVID-19: A review. Viruses. Epub ahead of print 2020. DOI: 10.3390/v12050527.

24. Bertolini A, van de Peppel IP, Bodewes FAJA, et al. Abnormal Liver Function Tests in Patients With COVID-19: Relevance and Potential Pathogenesis. Hepatology 2020; 72: 1864-1872.

25. Yazar H, Kayacan Y, Ozdin M. De Ritis ratio and biochemical parameters in COVID-19 patients. Arch Physiol Biochem 2020; 0: 1-5.

26. Wang S, Ma P, Zhang S, et al. Fasting blood glucose at admission is an independent predictor for 28-day mortality in patients with COVID-19 without previous diagnosis of diabetes: a multi-centre retrospective study. Diabetologia. Epub ahead of print 2020. DOI: 10.1007/s00125-020-05209-1.

27. Lippi G, South AM, Henry BM. Electrolyte imbalances in patients with severe coronavirus disease 2019 (COVID-19). Ann Clin Biochem 2020; 57: 262-265.

28. Bhatraju PK, Ghassemieh BJ, Nichols M, et al. Covid-19 in Critically III Patients in the Seattle Region - Case Series. N Engl J Med. Epub ahead of print 2020. DOI: 10.1056/nejmoa2004500.

29. Yuan X, Huang W, Ye B, et al. Changes of hematological and immunological parameters in COVID-19 patients. Int J Hematol. Epub ahead of print 2020. DOI: $10.1007 / \mathrm{s} 12185-020-02930-w$.

30. Ullah W, Basyal B, Tariq S, et al. Lymphocyte-to-C-Reactive Protein Ratio: A Novel Predictor of Adverse Outcomes in COVID-19. J Clin Med Res 2020; 12: 415-422.

31. Mina A, Besien K Van, Platanias LC. Hematological manifestations of COVID-19. Leuk Lymphoma 2020; 0: 1-9. 